\title{
Humanized Recommender Systems: State-of-the-art and Research Issues
}

Citation for published version (APA):

Tran, T. N. T., Felfernig, A., \& Tintarev, N. (2021). Humanized Recommender Systems: State-of-the-art and Research Issues. ACM Transactions on Interactive Intelligent Systems, 11(2), [9]. https://doi.org/10.1145/3446906

Document status and date:

Published: 01/07/2021

DOI:

$10.1145 / 3446906$

Document Version:

Publisher's PDF, also known as Version of record

Document license:

Taverne

Please check the document version of this publication:

- A submitted manuscript is the version of the article upon submission and before peer-review. There can be important differences between the submitted version and the official published version of record.

People interested in the research are advised to contact the author for the final version of the publication, or visit the DOI to the publisher's website.

- The final author version and the galley proof are versions of the publication after peer review.

- The final published version features the final layout of the paper including the volume, issue and page numbers.

Link to publication

\footnotetext{
General rights rights.

- You may freely distribute the URL identifying the publication in the public portal. please follow below link for the End User Agreement:

www.umlib.nl/taverne-license

Take down policy

If you believe that this document breaches copyright please contact us at:

repository@maastrichtuniversity.nl

providing details and we will investigate your claim.
}

Copyright and moral rights for the publications made accessible in the public portal are retained by the authors and/or other copyright owners and it is a condition of accessing publications that users recognise and abide by the legal requirements associated with these

- Users may download and print one copy of any publication from the public portal for the purpose of private study or research.

- You may not further distribute the material or use it for any profit-making activity or commercial gain

If the publication is distributed under the terms of Article $25 \mathrm{fa}$ of the Dutch Copyright Act, indicated by the "Taverne" license above, 


\title{
Humanized Recommender Systems: State-of-the-art and Research Issues
}

\author{
THI NGOC TRANG TRAN and ALEXANDER FELFERNIG, Institute of Software Technology, \\ Graz University of Technology \\ NAVA TINTAREV, Faculty of Science and Engineering, Maastricht University
}

Psychological factors such as personality, emotions, social connections, and decision biases can significantly affect the outcome of a decision process. These factors are also prevalent in the existing literature related to the inclusion of psychological aspects in recommender system development. Personality and emotions of users have strong connections with their interests and decision-making behavior. Hence, integrating these factors into recommender systems can help to better predict users' item preferences and increase the satisfaction with recommended items. In scenarios where decisions are made by groups (e.g., selecting a tourism destination to visit with friends), group composition and social connections among group members can affect the outcome of a group decision. Decision biases often occur in a recommendation process, since users usually apply heuristics when making a decision. These biases can result in low-quality decisions. In this article, we provide a rigorous review of existing research on the influence of the mentioned psychological factors on recommender systems. These factors are not only considered in single-user recommendation scenarios but, importantly, also in group recommendation ones, where groups of users are involved in a decision-making process. We include working examples to provide a deeper understanding of how to take into account these factors in recommendation processes. The provided examples go beyond single-user recommendation scenarios by also considering specific aspects of group recommendation settings.

CCS Concepts: • Information systems $\rightarrow$ Collaborative filtering; Information retrieval; Recommender systems; Social recommendation; • Human-centered computing $\rightarrow$ Human computer interaction (HCI); • Applied computing $\rightarrow$ Psychology;

Additional Key Words and Phrases: Recommender systems, group recommender systems, human decision making, decision biases, psychological factors, group dynamics

\section{ACM Reference format:}

Thi Ngoc Trang Tran, Alexander Felfernig, and Nava Tintarev. 2021. Humanized Recommender Systems: State-of-the-art and Research Issues. ACM Trans. Interact. Intell. Syst. 11, 2, Article 9 (June 2021), 41 pages.

https://doi.org/10.1145/3446906

\footnotetext{
The reviewing of this article was managed by associate editor Berkovsky, Shlomo.

The work presented in this article has been developed within the scope of the OpENREQ project (Intelligent Recommender and Decision Technologies for Community-Driven Requirements Engineering) no. 732463, funded by the European Commission - H2020 ICT Program.

Authors' addresses: T. N. T. Tran and A. Felfernig, Institute of Software Technology, Graz University of Technology, Inffeldgasse 16b/II, Graz, Austria, 8010; emails: \{ttrang, alexander.felfernig\}@ist.tugraz.at; N. Tintarev, Faculty of Science and Engineering, Maastricht University, Paul-Henri Spaaklaan 1, Maastricht, The Netherlands, 6229 EN; email: n.tintarev@maastrichtuniversity.nl.

Permission to make digital or hard copies of all or part of this work for personal or classroom use is granted without fee provided that copies are not made or distributed for profit or commercial advantage and that copies bear this notice and the full citation on the first page. Copyrights for components of this work owned by others than ACM must be honored. Abstracting with credit is permitted. To copy otherwise, or republish, to post on servers or to redistribute to lists, requires prior specific permission and/or a fee. Request permissions from permissions@acm.org. (c) 2021 Association for Computing Machinery.

2160-6455/2021/06-ART9 \$15.00

https://doi.org/10.1145/3446906
}

ACM Transactions on Interactive Intelligent Systems, Vol. 11, No. 2, Article 9. Publication date: June 2021. 


\section{INTRODUCTION}

Recommender systems are efficient tools that help to cope with information overload issues in many application domains [22]. These systems support a user's decision-making process and suggest items that fit his/her individual wishes and needs [54]. More recent research has shown that recommender systems do not only support decisions of single users but also group decisions. Example scenarios are choosing a restaurant to have dinner with colleagues or choosing a tourism destination for summer holidays with friends. For such activities, group recommender systems suggest items by aggregating the preferences of individual group members [47, 101].

Extensive research has been carried out to understand how recommender systems influence decision-making processes. Studies in this line of research have pointed out that the design and evaluation of recommender systems should take into account psychological factors beyond recommendation algorithms [36]. Inspired by this finding, psychology-enhanced recommender systems have been recently developed to improve the quality of recommendations. In the literature, there are four psychological factors that have been studied predominantly. These are personality (e.g., References [16, 55, 70, 93, 95, 171]), emotions (e.g., References [2, 90, 119, 137, 175]), social factors (group dynamics) (e.g., References [28, 34, 60, 101, 102, 113, 116, 123, 165, 172, 173]), and decision biases (e.g., References $[4,8,46,48,98,146-148,166])$. The sections of this article are organized in line with these four factors.

Personality can be described as a set of characteristics of a person that influence his/her cognition, emotion, and behavior in different situations [110]. In the recommendation context, personality can have an impact on item selection. For instance, in the music domain, Rentfrow and Gosling [128] investigated how the music preferences of users are related to their personality. The experimental results show that a reflective person with openness to experiences usually likes jazz and blues, whereas an energetic person with high degree of extroversion and agreeableness likes hip-hop and electronic music.

Besides personality, emotions have proven to be a relevant factor in recommender systems [161]. When interacting with a recommender system, a user tends to articulate his/her preferences combined with emotions. If his/her emotional needs are satisfied, then the complete fulfillment of his/her objective requirements is regarded as less important [61]. This aspect motivates the development of new recommendation approaches that take into account a user's emotions to improve prediction quality and increase his/her satisfaction with recommended items. In this context, researchers also investigate the relationship between personality and emotions. For instance, Tamir [150] performed user studies to figure out if there exist correlations between personality dimensions (e.g., neuroticism and extraversion) and emotional states. The results show that users with a high level of neuroticism tend to increase their level of worry. Users with a low level of extraversion are less inclined to have a high level of happiness.

In group recommendation scenarios, decision making can be influenced by group dynamics reflecting interactions among group members. Some examples thereof are social trust, where a group member tends to follow the opinion of other group members whom he/she loves or trusts [123]; conformity, where a group member is implicitly forced to follow the opinion of other group members [50]; fairness, where the preferences of group members are considered as far as possible [165]; and consensus, where group members try to agree on a solution [165]. Group dynamics also shows connections to other psychological factors such as personality and emotions. For instance, in group decisions where conflicts among group members' preferences arise, group members with different personality traits might behave differently when resolving conflicts. It could be the case that assertive users vote for a solution that satisfies their preferences, whereas cooperative users search for a solution that satisfies the preferences of others $[123,127]$. Also, the emotional states of group 
members can affect how they deal with conflict situations. A user with positive emotions (e.g., happiness, satisfaction, and amusement) tends to be cooperative and supportive. In contrast, a user with negative emotions (e.g., fear, anger, and sadness) tends to be selfish or less cooperative (see also Section 5.3). Besides, emotions also play a role in group decision scenarios where a group member's emotional state can affect the emotional state of other group members [50]. For instance, a user may not feel good if he/she knows that his/her friend is not enjoying the movie they are jointly watching (see also Section 5.2).

Finally, decision biases are triggered by decision heuristics (decision patterns) that can lead to suboptimal decision outcomes. For instance, belief biases make users too dependent on prior knowledge. Furthermore, omission biases make users omit information perceived as risky [143]. Decision biases depend on the perception and experience of a user and thus, influence his/her decisionmaking behavior. In the context of recommender systems, decision biases have been studied independently of other psychological factors such as personality, emotions, and group dynamics. Up to now, with a few exceptions, experimental results regarding the existence of relationships between the mentioned psychological factors and decision biases are still missing. Ramos [126] pointed out that personality plays a crucial role in determining the cognitive style of a user and hence has a strong impact on his/her decision-making behavior. Furthermore, personality traits are also responsible for the creation of cognitive biases.

Summarizing, if recommender systems are able to take into account psychological factors, then human decision processes can be simulated in a more realistic fashion [123]. This also helps to generate more accurate recommendations and increase users' satisfaction with recommended items. There exists research that provides an overview of the influence of decision-psychological phenomena on decision-making processes (e.g., References [30, 74, 97, 98]). Some contributions focus on decision biases and their potential impacts on preference construction [48, 98]. Others target at decision-making models and their importance for recommender system development [30]. In the mentioned studies, psychological factors are analyzed from the perspective of how users make daily choices and how recommender systems can support choice processes [74]. Different from related work, our work brings a broader view of how psychological factors affect recommender systems. We focus on factors that have recently attracted more attention in recommender systems research. These are personality, emotion, social factors (group dynamics), and decision biases. Knowledge about these factors is extremely important to further increase the decision support quality of recommender systems. In this article, we discuss related work and analyze potential applications of the mentioned factors in recommendation scenarios. The contributions of this article are the following:

(1) We provide selective working examples to increase the understanding of the role of psychological factors in recommender systems.

(2) Our discussions are not limited to single-user recommendation scenarios but also consider psychological factors that are especially relevant to group recommender systems.

(3) We point out open research issues related to the analysis and inclusion of psychological factors in recommender systems.

The remainder of the article is structured as follows: In Section 2, we present the methodology used as a basis for this article. In Section 3 and Section 4, we present different approaches to take into account users' personality and emotions in recommendation processes. Impacts of group dynamics on group decision making are discussed in Section 5. The influence of decision biases on recommender systems is analyzed in Section 6. Finally, we summarize open issues for future work in Section 7 and conclude the article with Section 8. 


\section{RESEARCH METHOD}

The basis of our analysis was a systematic bibliographic review of the existing literature on psychological factors in recommender systems [117, 144]. We collected relevant references using keywords such as "recommender systems," "human decision making," and "psychological factors." For a deeper look at psychological factors in recommender systems, we used additional keywords: "decision biases," "personality-based recommendation," "emotion-based recommendation," "group dynamics," and "social factors" when collecting references. We searched for existing publications in digital libraries such as ACM, ${ }^{1}$ Google Scholar, ${ }^{2}$ ResearchGate, ${ }^{3}$ Science Direct, ${ }^{4}$ and Springer. ${ }^{5}$ To ensure high-quality references, we first checked the title, abstract, keywords, conclusion, tables, and figures of the collected publications. Thereafter, we used the following filtering criteria: (i) conference/workshop proceedings, articles, and books/book chapters published by prestigious conferences/workshops, journals, and publishers; (ii) presenting detailed discussions on our research topic; and (iii) providing logical and reasonable findings related to the research topic. We excluded irrelevant publications that did not meet the filtering criteria and were published as technical reports or Master/Ph.D. dissertations. Based on the mentioned criteria, we identified 175 publications. Thereof, 71 papers have been published in well-known conferences/workshops such as ACM Conference on Recommender Systems (RecSys), User Modeling, Adaptation and Personalization (UMAP), Intelligent User Interfaces (IUI), World Wide Web (WWW), IEEE Tools with Artificial Intelligence (ICTAI), Intelligent Information and Database Systems, International Conference on Persuasive Technology, and International Conference on Social Informatics (SocInfo). We found 40 studies from various computer science journals such as ACM Transactions on Interactive Intelligent Systems (TIIS), IEEE Transactions on Knowledge and Data Engineering, IEEE Transactions on Fuzzy Systems, AI Magazine, Fournal of Expert Systems with Applications, Journal of Applied Science, fournal of Intelligent Information Systems (FIIS), and User Modeling and User-Adapted Interaction (UMUAI). Furthermore, we found 38 studies published in journals on psychology, marketing, and economics, such as $\mathrm{Hu}$ man Performance, Psychological Reports, Psychological Science, Genetic Psychology, Personality and Social Technology, Marketing, and Econometric and Society. Finally, we filtered out 26 books and book chapters from well-known publishers such as Cambridge University Press, Consulting Psychologists Press, John Wiley \& Sons, Oxford University Press, Plenum Press, Prentice-Hall, and Springer, which have been regarded as suitable for our study.

The most relevant aspects discussed in the identified literature can be organized along the topics of personality, emotions, group dynamics, and decision biases. The remainder of this article follows this identified categorization.

\section{THE INFLUENCE OF PERSONALITY ON RECOMMENDER SYSTEMS}

Many studies in the existing literature show the existence of a strong connection between a user's personality and his/her interests $[24,69,128]$. Therefore, integrating personality-related aspects into recommender systems can help to more precisely identify user preferences and thus enhance recommendation quality [110]. In this section, we first introduce a definition of personality and models to describe a user's personality traits. Thereafter, to boost recommendation outcomes and resolve open issues, we discuss some related recommendation approaches.

\footnotetext{
${ }^{1}$ https://dl.acm.org/.

${ }^{2}$ https://scholar.google.at/.

${ }^{3}$ https://www.researchgate.net/.

${ }^{4}$ https://www.sciencedirect.com/.

${ }^{5}$ https://link.springer.com/.
} 


\subsection{Personality and Relevant Models}

"Personality indicates a set of characteristics and qualities that forms a uniquely individual style of users in thinking, feeling, and behaving in different situations" [23, 110, 133]. In recommender systems, personality can be regarded as part of a user profile, which is domain-independent (i.e., does not change across different domains) and context-independent (i.e., does not change with time and location) [159]. User personality can be detected explicitly or implicitly [41]. Explicitly, the personality of a user can be detected by asking him/her to answer a list of personality-related questions that are well-established in psychology. For instance, the Ten Item Personality Inventory (TIPI) [62] offers a 10-item (question) measure of the Big Five dimensions for situations when simple measures are needed. The 44-item Big Five Inventory (BFI) questionnaire can be used to detect a user's personality traits according to the dimensions openness, conscientiousness, extroversion, agreeableness, and neuroticism [79]. Such explicit strategies require significant efforts of users to answer the related questionnaire. Besides, users might not tell the truth, or they are not able to explicate their personality traits correctly. For these reasons, implicit strategies can be applied to identify a user's personality by observing his/her behavioral patterns (e.g., review comments [70]). Alternatively, personality traits can also be predicted based on user information logs (e.g., images, content postings, and item preferences) stemming from personal web sites [99], music collections [128], and social networks [11, 45, 63, 87, 100, 107, 120, 121].

There exist various models that are commonly used in recommender systems to describe users' personality traits. Examples thereof are the RIASEC model [67], the Thomas-Kilman model [50, 122, 127, 155], and the Five-Factor model [124]. The RIASEC model uses six vocational interests to describe a user's personality: realistic (indicating a do-er), investigative (indicating a thinker), artistic (indicating a creator), social (indicating a helper), entrepreneurial (indicating a persuader), and conventional (indicating an organizer). The Thomas-Kilman model identifies two dimensions to characterize a user's personality: assertiveness and cooperativeness. Assertiveness is the degree to which a user tries to satisfy his/her own needs. Cooperativeness is the degree to which a user tries to satisfy other users' concerns. These two dimensions construct five conflict-handling modes: avoiding, accommodating, compromising, competing, and collaborating. The Five-Factor model is also called Big Five model, which helps to develop a better understanding of individual differences in personality [103]. The model consists of the five factors Openness (O), Conscientiousness (C), Extroversion (E), Agreeableness (A), and Neuroticism (N) [103, 160]. These factors are used to differentiate individuals with regard to emotional, interpersonal, experiential, attitudinal, and motivational styles [163]. Openness (O) describes the willingness of a user to try new activities. Conscientiousness (C) describes how a user controls, regulates, and directs his/her impulses. A conscientious user is more aware of his/her activities and better organized than an unconscientious user. Extraversion (E) describes a user who is social, outgoing, and talkative. Agreeableness (A) describes a user who is friendly and cooperative. Finally, Neuroticism $(\mathrm{N})$ indicates a tendency of experiencing negative feelings. A neurotic user is more likely to be anxious and over-scrutinized about his/her issues.

\subsection{The Influence of Personality on Recommender Systems}

3.2.1 Increasing Prediction Quality. Personality can help to explain why a user prefers one option over others. As a consequence, including personality in recommender systems can help users to better understand the reasons for a suggestion [110]. Recently, to enhance recommendation quality and user experience, some researchers have attempted to incorporate personality aspects into the recommendation process [16, 55, 93]. Lin and McLeod [93] introduced a temperamentbased filtering approach that incorporates human temperament into the recommendation process. A user's temperaments are first explored and learned for the representation and segmentation of 
Table 1. Example 1 - Formal Definitions of Segments $S_{1} . . S_{16}$ in the Information Space

\begin{tabular}{|c|c|c|c|}
\hline Segment & Definition & Segment & Definition \\
\hline$S_{1}$ & $D_{S J} \cap D_{S P} \cap D_{N T} \cap D_{N F}$ & $S_{9}$ & $D_{N T} \cap D_{N F}-D_{S J}-D_{S P}$ \\
\hline$S_{2}$ & $D_{S J} \cap D_{S P} \cap D_{N T}-D_{N F}$ & $S_{10}$ & $D_{S J} \cap D_{N F}-D_{S P}-D_{N T}$ \\
\hline$S_{3}$ & $D_{S J} \cap D_{S P} \cap D_{N F}-D_{N T}$ & $S_{11}$ & $D_{S P} \cap D_{N T}-D_{S J}-D_{N F}$ \\
\hline$S_{4}$ & $D_{S J} \cap D_{N T} \cap D_{N F}-D_{S P}$ & $S_{12}$ & $D_{S J}-D_{S P}-D_{N T}-D_{N F}$ \\
\hline$S_{5}$ & $D_{S P} \cap D_{N T} \cap D_{N F}-D_{S J}$ & $S_{13}$ & $D_{S P}-D_{S J}-D_{N T}-D_{N F}$ \\
\hline$S_{6}$ & $D_{S J} \cap D_{S P}-D_{N T}-D_{N F}$ & $S_{14}$ & $D_{N T}-D_{S J}-D_{S P}-D_{N F}$ \\
\hline$S_{7}$ & $D_{S J} \cap D_{N T}-D_{S P}-D_{N F}$ & $S_{15}$ & $D_{N F}-D_{S J}-D_{S P}-D_{N T}$ \\
\hline$S_{8}$ & $D_{N F} \cap D_{S P}-D_{S J}-D_{N T}$ & $S_{16}$ & $\emptyset$ \\
\hline
\end{tabular}

an information space. Thereafter, the learned temperament concept is employed to identify the most relevant information units to be presented as recommendations. Bologna et al. [16] proposed a context-aware recommendation approach that enables the adaptation of suggested e-commerce services based on a user's personality and the current interaction context. The proposed system allows the user to interact with e-commerce services in different contexts (e.g., while traveling or in a shopping mall). The system uses a neural network whose input is the user's personality profile described by the RIASEC model [67]. The network's output is the weights indicating matching scores between the current context and the e-commerce service that may be of interest to the user. Fernández-Tobías et al. [55] proposed a personality-based matrix factorization algorithm that exploits a user's personality as auxiliary information for the recommendation process (see also Section 3.2.2). The personality of a user is collected implicitly using a behavior-oriented approach that analyzes his/her positive feedback inferred from click-through data, browsing history, and item consumption counts. Although the experimental results of the mentioned approaches show specific improvements in terms of recommendation accuracy, more tests with real-life datasets are still needed also to increase the robustness of the proposed systems. Moreover, existing approaches often do not discuss how user personality information is collected. However, this information plays a crucial role in personality-aware recommendations.

Example 1. We choose the approach proposed by Lin and McLeod [93] to develop a working example that clearly shows how the personality of a user can be included in the recommendation process. As mentioned above, this approach proposes a two-phase recommendation process (learning phase and recommendation phase) to suggest information units to a user.

Learning phase: Given an information space of segments $\left(S_{1} . . S_{16}\right)$ and the personality of users classified into the four temperaments SJ (Sensing and fudging), SP (Sensing and Perceiving), NT (Intuiting and Thinking), and NF (Intuiting and Feeling). Each segment includes sets of information units liked and disliked by users depending on their temperaments (see Table 1). For instance, segment $S_{7}=D_{S J} \cap D_{N T}-D_{S P}-D_{N F}$ consists of information units liked by users with the temperaments $S J$ and $N T$, and disliked by users with the temperaments $S P$ and $N F$. In this context, an information unit is represented as a term vector in an $n$-dimensional space ( $n$ is the number of terms contained in an information unit). The weight of each term is computed by the TF-IDF (Term Frequency and Inverse Document Frequency) method [131].

Assume we have a list of four information units $\left(d_{a}, d_{b}, d_{c}, d_{d}\right)$ with temperament interest distributions as shown in Table 2. Each information unit can be clustered into segments based on its popularity. The popularity of an information unit $d$ within a temperament type $t$ is the conditional probability $P\left(\right.$ like $\left._{d} \mid t\right)$. The information unit $d$ is classified into segments $S_{i}(i \in[1 . .16])$ if its 
Table 2. Example 1 - Information Units $d_{a}, d_{b}, d_{c}, d_{d}$ and Corresponding Numbers of Users Who Liked/Disliked Each Information Unit According to a Specific Temperament Out of

$\{S J, S P, N T, N F\}[93]$

\begin{tabular}{|c|c|c|c|c|c|c|c|c|}
\hline \multirow{2}{*}{ Information unit } & \multicolumn{2}{|c|}{ SJ } & \multicolumn{2}{|c|}{ SP } & \multicolumn{2}{|c|}{ NT } & \multicolumn{2}{c|}{ NF } \\
\cline { 2 - 10 } & like & dislike & like & dislike & like & dislike & like & dislike \\
\hline$d_{a}$ & 0 & 467 & 0 & 214 & 0 & 161 & 0 & 158 \\
\hline$d_{b}$ & 102 & 365 & 17 & 197 & 59 & 102 & 0 & 158 \\
\hline$d_{c}$ & 160 & 307 & 8 & 206 & 71 & 90 & 0 & 158 \\
\hline$d_{d}$ & 84 & 383 & 50 & 164 & 101 & 60 & 55 & 103 \\
\hline
\end{tabular}

Table 3. Example 1 - Popularity of the Given Information Units

\begin{tabular}{|c|c|}
\hline Information unit & Popularity \\
\hline \multirow{4}{*}{$d_{a}$} & $P\left(\right.$ like $\left._{d_{a} \mid S J}\right)=0 /(0+467)=0$ \\
\hline & $P\left(\right.$ like $\left._{d_{a} \mid S P}\right)=0 /(0+214)=0$ \\
\hline & $P\left(\right.$ like $\left._{d_{a} \mid N T}\right)=0 /(0+161)=0$ \\
\hline & $P\left(\right.$ like $\left._{d_{a} \mid N F}\right)=0 /(0+158)=0$ \\
\hline \multirow{4}{*}{$d_{b}$} & $P\left(\right.$ like $\left._{d_{b} \mid S J}\right)=102 /(102+365)=\mathbf{0 . 2 2} \sqrt{ }$ \\
\hline & $P\left(\right.$ like $\left._{d_{b} \mid S P}\right)=17 /(17+197)=0.08$ \\
\hline & $P\left(\right.$ like $\left._{d_{b} \mid N T}\right)=59 /(59+102)=\mathbf{0 . 3 7} \sqrt{ }$ \\
\hline & $P\left(\right.$ like $\left._{d_{b} \mid N F}\right)=0 /(0+158)=0$ \\
\hline \multirow{4}{*}{$d_{c}$} & $P\left(\right.$ like $\left._{d_{c} \mid S J}\right)=160 /(160+307)=\mathbf{0 . 3 4} \sqrt{ }$ \\
\hline & $P\left(\right.$ like $\left._{d_{c} \mid S P}\right)=8 /(8+206)=0.04$ \\
\hline & $P\left(\right.$ like $\left._{d_{c} \mid N T}\right)=71 /(71+90)=\mathbf{0 . 4 4} \sqrt{ }$ \\
\hline & $P\left(\right.$ like $\left._{d_{c} \mid N F}\right)=0 /(0+158)=0$ \\
\hline \multirow{4}{*}{$d_{d}$} & $P\left(l i k e_{d_{d} \mid S J}\right)=84 /(84+383)=\mathbf{0 . 1 8} \sqrt{ }$ \\
\hline & $P\left({ }^{2} i k e_{d_{d} \mid S P}\right)=50 /(50+164)=\mathbf{0 . 2 3} \sqrt{ }$ \\
\hline & $P\left(\right.$ like $\left._{d_{d} \mid N T}\right)=101 /(101+60)=\mathbf{0 . 6 3} \sqrt{ }$ \\
\hline & $P\left(\right.$ like $\left._{d_{d} \mid N F}\right)=55 /(55+103)=\mathbf{0 . 3 5} \sqrt{ }$ \\
\hline
\end{tabular}

An information unit is only classified into a segment with a specific temperament if its popularity value is above the threshold $\theta=0.10$. The numbers with $\sqrt{ }$ represent popularity values passing $\theta$.

popularity is greater than a pre-defined threshold $\theta$. This threshold is usually fixed to achieve a certain confidence level for the segment [93] (in this example, the threshold $\theta=0.10$ ). The information units' popularity values are calculated as shown in Table 3. Based on these results, we can specify a set of pairs (information unit, segment) showing segments where the information units belong to: $\left(d_{a}, S_{16}\right),\left(d_{b}, S_{7}\right),\left(d_{c}, S_{7}\right)$, and $\left(d_{d}, S_{1}\right)$.

Recommendation phase: Based on the outcomes of the learning phase, examples of recommendation scenarios are the following:

Scenario 1 - Recommending information units according to a user's temperaments: Given a user with the temperaments $S J$ and $N T$, information units in the segments that match the user's temperaments will be chosen. For instance, $d_{b}$ and $d_{c}$ in the segment $S_{7}$ are recommended to the user, since this segment is liked by users with the temperaments $S J$ and NT (see Table 1 ).

Scenario 2 - Recommending information units according to a user's interest key terms: Given a user $U$ with interest key terms as shown in Table 4 and corresponding (TF*IDF) weights as shown in Table 5, the similarity between user $U$ and an information unit $V$ is calculated using the 
Table 4. Example 1 - Key Terms of the Information Units $d_{a}, d_{b}, d_{c}, d_{d}$ and Interest Key Terms of the User

\begin{tabular}{|c|c|}
\hline Information units & Key terms \\
\hline$d_{a}$ & recommender, evaluation, social \\
\hline$d_{b}$ & practical, recommender, algorithms \\
\hline$d_{c}$ & statistics, recommender, methods \\
\hline$d_{d}$ & recommendation, automated, algorithms \\
\hline interest key terms of the user & recommender, algorithms, evaluation \\
\hline
\end{tabular}

Table 5. Example 1 - Key Terms of the Information Units (Presented in Table 4) and Corresponding Weight Values Computed Using TF-IDF [131]

\begin{tabular}{|c|c|c|c|c|c|}
\hline \multirow{2}{*}{$\begin{array}{c}\text { Key } \\
\text { terms }\end{array}$} & \multicolumn{5}{|c|}{ Weight (TF* $\mathbf{I D F )}$} \\
\cline { 2 - 6 } & $d_{a}$ & $d_{b}$ & $d_{c}$ & $d_{d}$ & user \\
\hline algorithms & 0 & 0.74 & 0 & 0.74 & 0.74 \\
\hline automated & 0 & 0 & 0 & 2.32 & 0 \\
\hline evaluation & 1.32 & 0 & 0 & 0 & 1.32 \\
\hline methods & 0 & 0 & 2.32 & 0 & 0 \\
\hline practical & 0 & 2.32 & 0 & 0 & 0 \\
\hline recommender & 0.32 & 0.32 & 0.32 & 0 & 0.32 \\
\hline recommendation & 0 & 0 & 0 & 2.32 & 0 \\
\hline social & 2.32 & 0 & 0 & 0 & 0 \\
\hline statistics & 0 & 0 & 2.32 & 0 & 0 \\
\hline
\end{tabular}

Cosine similarity (see Formula (1)). Similar to an information unit, a user vector is also represented as an $n$-dimensional vector, where $n$ is the number of interest key terms.

$$
\operatorname{sim}(U, V)=\frac{\sum_{i=1}^{n}\left(U_{i} \times V_{i}\right)}{\sqrt{\sum_{i=1}^{n} U_{i}^{2}} \times \sqrt{\sum_{i=1}^{n} V_{i}^{2}}}
$$

In the example, the similarity between the user $U$ and each information unit $d$ is calculated as follows: $\operatorname{sim}\left(U, d_{a}\right)=\mathbf{0 . 4 4} \sqrt{ }, \operatorname{sim}\left(U, d_{b}\right)=0.17, \operatorname{sim}\left(U, d_{c}\right)=0.02, \operatorname{sim}\left(U, d_{d}\right)=0.1$. The information unit $d_{a}$ is recommended to the user, since it achieves the highest similarity with the user interests.

Obviously, the approach mentioned in Scenario 2 is not a personality-aware recommendation. However, it can be applied in the context of the following scenario:

Scenario 3 - Recommending information units according to a user's temperaments and interest key terms: Given a user $U$ with the temperaments $S J$ and NT and his/her interest key terms as mentioned in Table 4, the recommendation can be generated using the results in Scenarios 1 and 2. According to the user's temperaments, the segment $S_{7}$ is chosen for the recommendation (see Scenario 1). Besides, $S_{7}$ consists of two information units $d_{b}$ and $d_{c}$, where $\operatorname{sim}\left(U, d_{b}\right)>\operatorname{sim}\left(U, d_{c}\right)$ (see Scenario 2). Therefore, the information unit $d_{b}$ of segment $S_{7}$ is recommended to the user.

3.2.2 Resolving Cold-start Problems. A collaborative filtering recommender system might face a new user cold-start problem where it cannot generate personalized recommendations for a new user 
Table 6. Example 2 - Four Users $\left\{u_{1}, u_{2}, u_{3}, u\right\}$ Whose Personality Traits Are Represented According to the Five-Factor Model [103]: Openness (O), Conscientiousness (C), Extroversion (E), Agreeable (A), and Neuroticism $(\mathrm{N})$

\begin{tabular}{|c|c|c|c|c|c||c|}
\hline User & $\mathbf{O}$ & $\mathbf{C}$ & $\mathbf{E}$ & $\mathbf{A}$ & $\mathbf{N}$ & Average of personality-trait values \\
\hline$u_{1}$ & 0.85 & 0.61 & 0.12 & 0.78 & 0.33 & 0.538 \\
\hline$u_{2}$ & 0.27 & 0.5 & 0.88 & 0.91 & 0.47 & 0.606 \\
\hline$u_{3}$ & 0.59 & 0.87 & 0.36 & 0.8 & 0.55 & 0.634 \\
\hline new user $(u)$ & 0.75 & 0.59 & 0.68 & 0.75 & 0.23 & 0.6 \\
\hline
\end{tabular}

The value for each factor is normalized to the range of [0..1].

without rating history $[5,55]$. In this context, the lack of user information (e.g., ratings, purchased items, browsing history) causes recommendations with a low correlation with the user's interests [43]. One common solution is to apply hybrid recommendation approaches that combine content information and item ratings [21]. Another solution is to use demographic information (e.g., age, gender, and educational background) to calculate the similarity between two users [115].

Recently, novel approaches considering the personality of users have been proposed in recommender systems. These are helpful for solving the new user problem and increasing the accuracy of recommendations. For instance, the similarity between two users can be calculated using a Pearson correlation coefficient with the inclusion of the users' personality descriptors [70]. The personality of a user $u$ is represented as an $n$-dimensional vector $p_{u}=\left(p_{u}^{1}, p_{u}^{2}, \ldots, p_{u}^{n}\right)^{T}$, in which each dimension indicates a personality trait of the user. According to the Five-Factor Model [103], $p_{u}$ is a five-dimensional vector representing five personality traits: Openness $(\mathrm{O})$, Conscientiousness $(\mathrm{C})$, Extroversion (E), Agreeableness (A), and Neuroticism (N). Besides, $\overline{p_{u}}$ and $\overline{p_{v}}$ indicate the average of personality-trait values of users $u$ and $v$. The personality similarity between $u$ and $v-\operatorname{simp}(u, v)$ is calculated using Formula (2).

$$
\operatorname{simp}(u, v)=\frac{\sum_{k}\left(p_{u}^{k}-\overline{p_{u}}\right) \times\left(p_{v}^{k}-\overline{p_{v}}\right)}{\sqrt{\sum_{k}\left(p_{u}^{k}-\overline{p_{u}}\right)^{2} \times \sum_{k}\left(p_{v}^{k}-\overline{p_{v}}\right)^{2}}}
$$

Example 2. For a better understanding, we exemplify the mentioned approach. Given a new user $u$ and a list of three users $\left\{u_{1}, u_{2}, u_{3}\right\}$ with personality traits as shown in Table 6 , the personality similarities between user $u$ and the other users are calculated using Formula (2): $\operatorname{simp}\left(u, u_{1}\right)=\mathbf{0 . 4 7}$, $\operatorname{simp}\left(u, u_{2}\right)=0.28$, and $\operatorname{simp}\left(u, u_{3}\right)=0.14$. In terms of personality, user $u_{1}$ is the nearest neighbor of user $u$. Thus, items liked by $u_{1}$ can be recommended to $u[24,128]$.

$\mathrm{Hu}$ et al. $[69,70]$ proposed a hybrid approach including both rating-based and personalitybased recommendations. In this approach, the similarity between two users $u$ and $v$ is calculated using Formula (3), where $\operatorname{simr}^{\prime}(u, v)$ is the item-based similarity and $\operatorname{simp}(u, v)$ represents the personality-based similarity between $u$ and $v$. Parameter $\alpha$ is used to control the influence of ratingbased similarity on the final similarity measurement.

$$
\operatorname{sim}(u, v)=\alpha \times \operatorname{simr}^{\prime}(u, v)+(1-\alpha) \times \operatorname{simp}(u, v)
$$

In the same line of research, Fernández-Tobías et al. [55] proposed three approaches to address the new user problem. The most useful preference information is determined based on the personality of users. This information is the premise to generate the most relevant recommendations for a new user. The underlying idea of these approaches is discussed in the following (for more details, we refer to Reference [55]):

(1) Personality-based matrix factorization: This approach extends classical matrix factorization by incorporating additional latent feature vectors that represent personality aspects. The training 


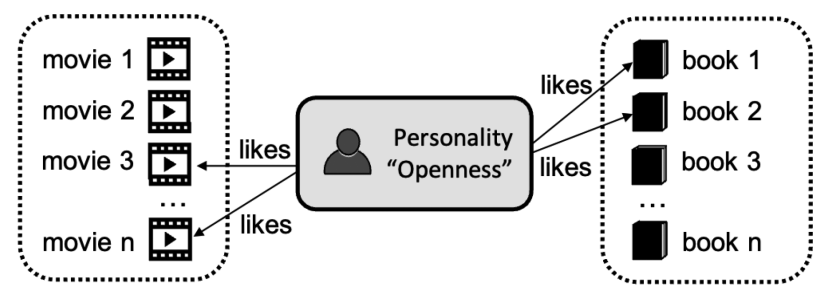

Fig. 1. An example of cross-domain recommendation using personality information [68].

procedure is based on the alternating least squares technique [71]. Using this approach, missing values can be partially compensated with the personality information of users. This is beneficial if the target user has no rating history.

(2) Personality-based active learning: This approach utilizes personality information to find and elicit the most informative user ratings. Instead of using item ratings as in traditional active learning models, positive-only feedback (e.g., likes, click-through data, and item consumption counts) is exploited to generate recommendations. Recommendations based on this approach receive more "likes" from completely new users compared to baseline methods.

(3) Personality-based cross-domain recommendation: This approach utilizes personality information to better predict user preferences from auxiliary source domains. It helps to compensate for missing user information in the target domain. Experimental results in different domains (e.g., movies, music, and books) show that this approach resolves the new-user problem very well, especially in cases where new users did not provide any ratings.

A similar approach of employing personality information in cross-domain recommendation was proposed by $\mathrm{Hu}$ [68]. The general idea is the following: "If a user wants to receive recommendations of two products from different domains (e.g., movies and books), then the system needs the user's rating data for both products." In previous approaches, rating data is usually used separately and could not be transformed across different domains. For instance, movie domain ratings could not be used in the book domain and vice versa. However, personality information can be utilized to link different types of products.

Given, for example, the two domains of movies and books connected by the personality trait Openness, the user can get a personalized recommendation using one of the following two approaches (see also Figure 1):

-Approach 1: Since the system knows that the user belongs to the personality trait Openness, it can recommend to him/her movie $_{3}$ and movie $_{n}$ from the movie domain, and book 1 and book $k_{2}$ from the book domain based on the correlation between personality and domain items.

-Approach 2: The system does not know the user's personality in advance. It first infers the user's personality traits according to his/her preferences in the movie domain. Based on the inferred personality, the system recommends book $k_{1}$ and $b o o k_{2}$ from the book domain.

The studies mentioned in this subsection introduce different ways to tackle cold-start problems by incorporating user personality into the recommendation process. These approaches have proven to outperform traditional recommendation techniques in terms of effectiveness [55, 68]. However, the results are not completely convincing, since only datasets from specific domains (e.g., music, movie, and book) have been used, which might not sufficiently reflect the proposed methods' real performance. Therefore, further tests with real-life datasets are needed to further evaluate personality-based recommendation approaches.

3.2.3 Diversifying Recommendations. In recommender systems, the importance of diversity has been discussed with regard to two aspects: "counteracting over-fitting" and "increasing user 
satisfaction" [89]. Recommendation diversity is also a solution to counteract "popularity biases" in recommender systems (popular items are recommended more often than less popular ones [1]).

Many studies have recently been performed to investigate whether users with different personalities have different needs regarding diversification. For instance, Ferwerda and Schedl [56] determined users' personalities based on the Five-Factor model [103] and used these as user models to investigate preferred diversification levels. The authors pointed out that conscientious users prefer a higher degree of diversification, whereas agreeable users show a medium level of diversification needs. Wu et al. [171] diversified an item recommendation list using a greedy re-ranking approach performed in two steps. In Step 1, the algorithm predicts the preferences of a user $u$ for an un-experienced item $i\left(\operatorname{Score}_{\operatorname{Pref}}(u, i)\right)$ based on his/her personality. Particularly, the user's preference for item $i$ can be predicted based on the rating profiles of her/his neighbors with similar personality traits. The predicted preference score of user $u$ for each item is saved in an item set $S$ and sorted in descending order $(S$ denotes a candidate item set $($ size $=n))$. In Step 2, the algorithm re-ranks the items in $S$ to achieve a top-N recommendation list $T$ that meets the user's diversification preferences. $T$ denotes the re-ranked list including $N$ items $(N<=n)$ that user $u$ will finally receive. To determine this list, for each item $i \in S$, the personalized diversification score Score $_{\text {PersonalizedDiv }}(u, i)$ is calculated based on the personality of user $u$. This score is then combined with the un-experienced item score $\operatorname{Score}_{\text {Pref }}(u, i)$ to achieve the final score $\left(\right.$ Score $\left._{\text {inal }}\right)$ (see Formula (4)).

$$
\operatorname{Score}_{\text {final }}(u, i)=\beta \times \operatorname{Score}_{\text {Pref }}(u, i)+(1-\beta) \times \operatorname{Score}_{\text {PersonalizedDiv }}(u, i)
$$

In this context, $\beta$ is used to balance two types of preferences-predicted preference and diversification preference. The value of $\operatorname{Score}_{\text {final }}(u, i)$ is used to select items for $T$, which "optimizes" the tradeoff between a user's preference for an item and his/her diversification preferences. This way, items with the best balance between accuracy and personalized diversification are added to the recommendation list.

Based on experimental results, $\mathrm{Wu}$ et al. [171] show that personality-based greedy re-ranking outperforms non-diversity-oriented methods (e.g., rating-based collaborative filtering) in terms of accuracy and diversification preference. One drawback of this approach is that it is time-consuming when collecting the personality of users using the 44-item BFI questionnaire. Nevertheless, this work has important implications for recommender systems: (1) The diversification preferences of a user can be inferred from his/her personality traits; (2) personality assists recommender systems in better understanding the inherent preferences of a user and helps to provide higher-quality recommendation services.

The discussed recommender systems primarily focus on analyzing personality factors in singleattribute item domains. However, in cases where items are characterized by different attributes, users with different personality characteristics might have different diversification needs according to item attributes. Inspired by the approach of $\mathrm{Wu}$ et al. [171], Lu and Tintarev [95] proposed a personality-aware recommender system in the song domain, which considers the relationship between personality traits and song attributes. In this approach, an objective function is used to select a candidate item for the diversified recommendation list. The candidate item must have the lowest objective function value, meaning that the item manages to maximize the degree of user preference satisfaction.

Example 3. For demonstration purposes, we choose the approach proposed by Lu and Tintarev [95]. This approach provides a method that incorporates personality information into the recommendation process to generate a diversified recommendation list. 
Table 7. Example $3-R=\left\{s_{1} . . s_{8}\right\}$ Represents the List of Songs Recommended to User $u$ without Taking into Account Diversification Preferences

\begin{tabular}{|c|c|c|c|c|c|c|c|c|}
\hline & $s_{1}$ & $s_{2}$ & $s_{3}$ & $s_{4}$ & $s_{5}$ & $s_{6}$ & $s_{7}$ & $s_{8}$ \\
\hline genre & pop & pop & pop & rock & rock & country & dance & classic \\
\hline artist number & 1 & 1 & 3 & 3 & 2 & 1 & 3 & 1 \\
\hline key & 6 & 6 & 5 & 6 & 6 & 7 & 5 & 7 \\
\hline
\end{tabular}

Each song is characterized by the three attributes "genre," "artist number," and "key." The attribute "key" is an audio feature of a song, which can be figured out by using a key signature. The key of a song describes the number of sharps and flats in the key signature. For instance, $k e y\left(s_{3}\right)=5$ indicates that the number of sharps/flats in the key signature is 5 .

Table 8. Example 3 - Personality Traits of User $u$, Where $E=$ Extroversion, $A=$ Agreeableness, $C=$ Conscientiousness, $E S=$ Emotional Stability (the Opposite of Neuroticism Mentioned in Section 3.1), and $O=$ Openness

\begin{tabular}{|c|c|c|c|c|c|}
\hline & E & A & C & ES & O \\
\hline personality traits & medium low & high & low & low & medium low \\
\hline mapping scores & 0.4 & 0.8 & 0.2 & 0.2 & 0.4 \\
\hline
\end{tabular}

The traits are measured on a four-level scale (see row 2) that are converted to numeric scores (see row 3). Based on experimental results, Lu and Tintarev [95] pointed out that the personality traits $E, A$, and ES have the relationships with the attributes "key," "artist number," and "genre," respectively. The remaining traits $C$ and $O$ do not show significant correlations with the song attributes.

Assume $R=\left\{s_{1} . . s_{8}\right\}$ is the list of songs recommended to an active user $u$ without taking into account diversification preferences. This information is summarized in Tables 7 and 8 . Now, a diversified recommendation list $D$ (we assume that the length of $D$ is $N=2$ ) can be derived.

A diversified recommendation list $D$ can be derived using the following steps:

Step 1: Assign the first song $s_{1} \in R$ for the first element of $D$, i.e., $D[1]=R[1]$.

Step 2: Find the next candidate song for $D$ using an objective function. The objective function value of each song $s_{i} \in R$ (which has not been chosen for $D$ so far) is calculated using Formula (5).

$$
\operatorname{Obj}\left(s_{i}, D\right)=\operatorname{Rank}\left(s_{i}, R\right) \times(1-\lambda)+\lambda \times \operatorname{Rank}\left(\operatorname{Div}_{\text {overall }}\left(s_{i}, D\right)\right)
$$

In this context, $\operatorname{Rank}\left(s_{i}, R\right)$ represents the rank of song $s_{i} \in R$. The recommended songs in $R$ are ranked in descending order, i.e., $\operatorname{rank}\left(s_{1}, R\right)=1$ and $\operatorname{rank}\left(s_{8}, R\right)=8$. The parameter $\lambda$ controls the tradeoff between similarity and diversity, ensuring that an item selected for $D$ is not so far from the user's preferences. This parameter is determined based on the relationship between personality traits and the overall diversity degree Div overall $\left(s_{i}, D\right)$. The experimental results in Reference [95] show that the personality trait ES (Emotional Stability) has a positive correlation with the overall diversity degree. Therefore, $\lambda$ is adjusted according to the user's Emotional Stability level.

We assume that the overall diversity values Div overall $\left(s_{i}, D\right)$ of songs $s_{i} \in R, i \in[2 . .8]$ (that have not been chosen for $D$ so far) are shown as entries of column 2, Table 9. These values have to be ranked in descending order before calculating the objective function value. The outcome of applying the objective function for each $s_{i} \in R$ is the following:

$$
\begin{aligned}
& \operatorname{Obj}\left(s_{2}, D\right)=3 \\
& \operatorname{Obj}\left(s_{3}, D\right)=2.8 \sqrt{ } \\
& \operatorname{Obj}\left(s_{4}, D\right)=3.8 \\
& \operatorname{Obj}\left(s_{5}, D\right)=4.6 \\
& \operatorname{Obj}\left(s_{6}, D\right)=5.8
\end{aligned}
$$


Table 9. The Overall Diversity Values $\operatorname{Div}_{\text {overall }}\left(s_{i}, D\right)$ of the Songs $s_{i} \in R(i=\{2 . .8\})$, Which Have Not Been

Selected So Far

\begin{tabular}{|c|c|c|}
\hline & Div $_{\text {overall }}\left(s_{i}, D\right)$ & $\operatorname{Rank}\left(\operatorname{Div}_{\text {overall }}\left(s_{i}, D\right)\right)$ \\
\hline$s_{2}$ & 0 & 7 \\
\hline$s_{3}$ & 0.67 & 2 \\
\hline$s_{4}$ & 0.56 & 3 \\
\hline$s_{5}$ & 0.56 & 3 \\
\hline$s_{6}$ & 0.33 & 5 \\
\hline$s_{7}$ & 0.77 & 1 \\
\hline$s_{8}$ & 0.33 & 5 \\
\hline
\end{tabular}

These values have to be ranked in descending order before applying the objective function.

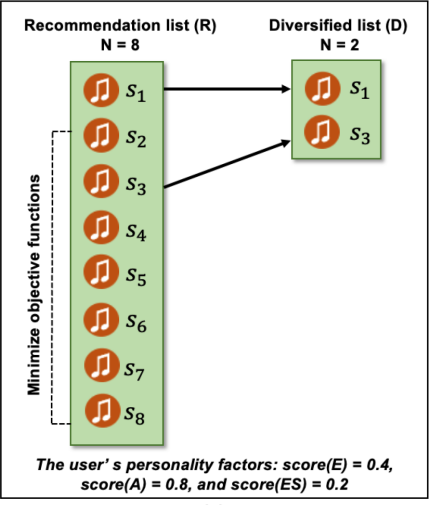

(a)

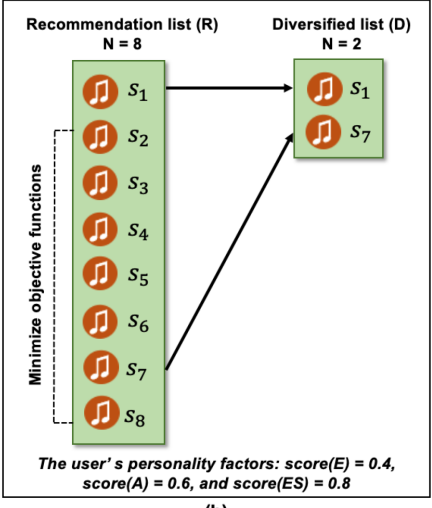

(b)

Fig. 2. Example 3 - Diversified lists $D$ generated from the same recommendation list $R$ based on personality traits [95]. Scenarios (a) and (b) show that users with different personality traits have different preferences regarding recommendation diversity.

$\operatorname{Obj}\left(s_{7}, D\right)=5.8$

$\operatorname{Obj}\left(s_{8}, D\right)=7.4$

Step 3: Include $s_{3}$ in $D$, since $\operatorname{Obj}\left(s_{3}, D\right)$ is minimal. At this point, length $(D)=2$ and the process ends. $D=\left\{s_{1}, s_{3}\right\}$ is the diversified recommendation list for user $u$ (see Figure 2(a)).

Users with different personality traits have different diversification needs [56]. For instance, given the same recommendation list $R$ and a user $v$ with the personality traits $\operatorname{score}(E)=0.4$, $\operatorname{score}(A)$ $=0.6$, and $\operatorname{score}(E S)=0.8$, we can specify the diversified recommendation list $D$ for user $v$ as follows: $D=\left\{s_{1}, s_{7}\right\}$ (see Figure 2(b)). A similar study was performed by Chen et al. [32], who focused on the investigation of the influence of Openness on users' diversification preferences. The authors show that users with a high level of Openness prefer having a more diverse set of recommended items compared to users with a low level of Openness.

Although Lu and Tintarev [95] provided an effective solution for generating diversified recommendations for multi-attribute item problems, it seems that this solution strongly depends on the extracted relationships between personality traits and item attributes. With a pilot study of 148 participants, the authors found out that the personality traits Extroversion, Agreeableness, and Emotional Stability have relationships with the song attributes "key," "artist number," and "genre," 


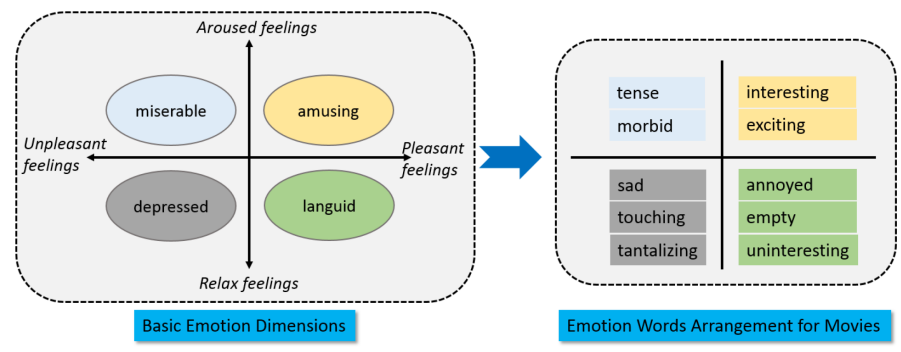

Fig. 3. Basic Emotion Dimensions [42] and Emotion-words Arrangement for movies [142].

respectively. However, with a different set of participants, the finding could change, which leads to different outcomes of the diversified recommendation. Thus, to effectively apply this approach, the relationships between personality traits and item attributes need to be analyzed in further detail.

\section{THE INFLUENCE OF EMOTIONS ON RECOMMENDER SYSTEMS}

Besides personality, emotions play an essential role in the decision making-behavior of users. Emotions are volatile and difficult to describe [162]. There exist two dominant approaches to model emotions: categorical and dimensional [80]. The categorical approach represents emotions via a set of distinct emotional categories, e.g., happiness, sadness, anger, surprise, fear, and interest [73]. The dimensional approach conceptualizes emotions based on their approximate placement in a broad and continuous multi-dimensional space, where each dimension represents the emotion's quality $[80,162]$. The most commonly used dimensions are valence/pleasure, arousal/activation, and dominance, which are constructed in different forms, such as one-dimensional arousal models, two-dimensional models (e.g., arousal - pleasure [130] and basic emotion dimensions [42]-see also Figure 3), or three-dimensional models (e.g., energy arousal - tense arousal - valence [134]). The most popular version is the circumplex model proposed by Russell [130]. This model consists of a two-dimensional and circular structure, representing the dimensions arousal and pleasure [80].

Many psychological and sociological studies show that emotions can be characterized as a kind of context strongly connected to users' preferences. Thus, emotions can direct users toward decisions that differ from those generated in a "more" rational mental process $[104,161]$. As a consequence, preferences can be regarded context-dependent, and emotions are a key factor that has an impact on the "chosen" context. For instance, a user listens to electronic music when he/she is happy and blue/jazz music when he/she is feeling depressed. For this reason, recommender systems should be aware of the emotional state of users and make use of such emotion-related information. To improve recommendation outcomes, researchers have proposed various approaches considering emotions in the recommendation process. Most of these studies focus on answering the following two research questions:

(1) How to detect users' emotions in recommendation contexts?

(2) How to take into account emotions in recommendation algorithms to improve recommendation performance and increase user satisfaction with recommended items?

\subsection{How to Detect Emotions?}

Emotions can be detected either explicitly or implicitly. Explicitly, the emotions of a user can be detected using self-assessment methods where he/she is asked to answer questions designed to measure different emotion-related features. For instance, Bradley and Lang [18] proposed a picture-oriented questionnaire, the so-called Self-Assessment Manikin (SAM), to measure three features of an emotional response (valence, arousal, and dominance). Implicitly, emotions can be 
detected using two approaches: physical signals and internal signals [140]. The first approach analyzes changes regarding a user's physical signals through multiple modalities (e.g., facial expressions, body movements, gestures, and speech) that have shown to be associated with specific emotions [27]. The second approach is to use physiological signals (e.g., electroencephalogram, temperature, electrocardiogram, galvanic skin response, respiration) to detect the user's emotional states. For this approach, a system consisting of sensors and neuron devices is used to analyze changes in physiological signals [140].

The mentioned emotion detection methods have advantages and disadvantages. With explicit approaches, although the accuracy of collected emotions can not be warranted (since the user may not tell the truth or face some difficulties in expressing his/her emotions), the application to recommender systems is relatively easy. In contrast, implicit approaches can provide more precise results compared to explicit approaches. However, they are quite expensive to be applied in recommender systems. Consequently, finding efficient emotion detection methods that support a tradeoff between emotion detection accuracy and applicability in recommender systems is, to some extent, still an open issue.

\subsection{How to Integrate Emotions in Recommendation Algorithms?}

Emotions can be integrated into recommendation algorithms as a contextual parameter. Zheng et al. [175] performed a study to explore how to use emotions to improve recommendation quality. In their approach, emotion-related information is integrated into two classes of context-aware recommendation algorithms: context-aware splitting (CAS) and differential context modeling (DCM). In $C A S$, the authors introduced two approaches: item splitting and user splitting. Item splitting finds a contextual condition to split each item. The underlying idea of this approach is that an item experienced in different contextual conditions can be split into different items [13]. Similarly, user splitting considers one user as different users if he/she demonstrates significantly different preferences across contexts. DCM presents a way to determine which emotion contexts are influential for which algorithm components. The authors proved that both of the proposed approaches help to increase recommendation quality.

Another approach is to explore a user's emotions based on music/songs that he/she listens to. For instance, Kuo et al. [90] and Shan et al. [137] proposed a music-emotion model to infer a user's emotions from consumed film music. The model consists of three components: (1) film music emotion detection, (2) film music feature extraction, and (3) emotion-based music recommendation. The first component detects the film music emotion from film videos that provide useful cues (e.g., caption, speech, sound effect, and visual features). The second component extracts features that have strong effects on emotions (e.g., chord, melody, tempo, and rhythm). Third, a Mixed Media Graph [114] and a graph-based approach are utilized and modified to discover associations between emotions and extracted music features. The associations are then applied to generate music recommendations. Experimental results show that the proposed model generates music recommendations with $85 \%$ accuracy on an average. In the movie domain, Song et al. [142] proposed an emotion-words selection approach to derive users' emotions for movies. For this approach, the authors first selected some movies from the "Korean Film Council" database, which covers various movie tastes of users. Thereafter, 10 emotional words proposed by Lee and Jeong [92] were adjusted to make them suitable for the movie domain. These emotional words were arranged according to Basic Emotion Dimensions [42] (see Figure 3) and then integrated into a questionnaire for the purpose of emotion detection. Experimental results show that the Emotion-words Arrangement [142], which is represented based on the Basic Emotion Dimensions, is appropriate for reflecting users' emotions and predicting users' movie preferences. 
Abdul et al. [2] developed an Emotion-Aware Personalized Music Recommendation System (EPMRS) where emotions and other dimensions such as time and location were integrated in the recommendation process. The authors used weighted matrix factorization [71] to extract latent features from user-to-song relationships. The ratings for the songs that the user did not listen to up to now were predicted. When generating recommendations, the system considers additional information: emotion (e.g., happy, normal, sad, and surprised), listening time (e.g., morning, noon, afternoon, evening, and midnight), and current location (e.g., home, workplace, and others). The user is able to inform the system about his/her current emotion through four emotion types (happy, normal, sad, and surprised). Based on the articulated emotions, EPMRS recommends songs that meet the user's emotions in the current situation. A user can adapt his/her emotions anytime, and EPMRS is able to recommend songs according to the user's current emotional state. This way, song recommendations determined by EPMRS can trigger a higher user satisfaction compared to traditional recommender systems (e.g., content-based recommender systems) [2].

Example 4. To develop an illustrative example, we sketch the approach proposed by Abdul et al. [2]. This approach shows how emotional information can be taken into account in recommender systems. The recommendation process consists of the following steps:

Step 1 - Specify a user-to-song interest matrix: Let $U=\left\{u_{1}, u_{2}, \ldots, u_{n}\right\}$ denote the set of $n$ users, $S=\left\{s_{1}, s_{2}, \ldots, s_{m}\right\}$ be the set of $m$ songs, and $N$ with entries $n_{i, j} \in Z^{+}(i \in[1 \ldots n], j \in$ $[1 \ldots m])$ be the user-to-song interest matrix. Each entry of $N$ represents a user's preference for a song, i.e., dislike (0) or like ( 1 to 5 stars). The entry "-" indicates that a user has not listened to a song. $N$ represents the relationship between users and songs in a specific context characterized by emotion, time, and location. For instance, $N(\mathrm{sad}$, afternoon, work place) represents users' song preferences under the contextual conditions "being sad" and "listening to music in the afternoon at the workplace."

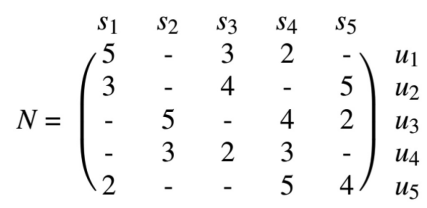

Step 2 - Specify a matrix representing the latent features of the songs: Let matrix $M$ with entries $m_{j, k} \in[0,1](j \in[1 \ldots m], k \in[1 \ldots v])$ represent the latent features of the songs $(v$ is the number of the latent features). In this example, each song has four latent features $\left(f_{1} \ldots f_{4}\right)$ and $M$ is represented as the matrix shown below. For instance, $M_{1, j}=[0,1,0,1]$ indicates that $s_{1}$ can be related to the latent features $f_{2}$ and $f_{4}$, but not the features $f_{1}$ and $f_{3}$.

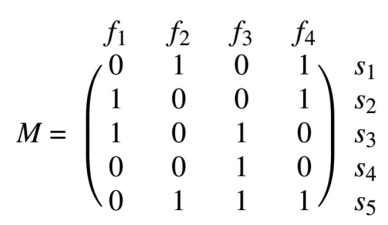

Step 3 - Specify a matrix representing the relationship between users and latent features: The matrices $N$ and $M$ are used to generate a new matrix $Q$ with entries $q_{i, k} \in Z^{+}(i \in$ $[1 \ldots n], k \in[1 \ldots v])$. Each entry $q_{i, k}$ represents the frequency of a feature to appear in the songs listened to by a user. In this example, $Q$ can be determined as shown in the following: For instance, $q_{4,1}=2$ indicates that feature $f_{1}$ was regarded as relevant "twice" in the songs listened to by user $u_{4}$. This entry is specified as follows: User $u_{4}$ listened to the songs $s_{2}, s_{3}$, and $s_{4}$ (see matrix $N$ ). Based on matrix $M, f_{1}$ is the latent feature of $s_{2}$ and $s_{3}\left(m_{2,1}=m_{3,1}=1\right)$, but not the latent feature 
of $s_{4}\left(m_{4,1}=0\right)$. Consequently, the frequency of feature $f_{1}$ to appear in the rated songs of user $u_{4}$ is $q_{4,1}=1+1+0=2$.

$$
Q=\left(\begin{array}{cccc}
f_{1} & f_{2} & f_{3} & f_{4} \\
1 & 1 & 2 & 1 \\
1 & 2 & 2 & 2 \\
2 & 1 & 2 & 2 \\
0 & 0 & 2 & 1 \\
0 & 2 & 2 & 2
\end{array}\right) \begin{aligned}
& u_{2} \\
& u_{3} \\
& u_{4} \\
& u_{5}
\end{aligned}
$$

Step 4 - Specify the weight of latent features: Latent features are assumed to have different impacts (i.e., weights) on users' preferences. Some features have a higher impact than others. Abdul et al. [2] used the TF-IDF approach [170] as the user feature frequency $F_{U}\left(f_{k}\right)$ and the inverse user feature frequency $F_{U}^{-1}\left(f_{k}\right)$ to determine important features. ${ }^{6}$ A matrix $W$ with entries $w_{i, k} \in Z^{+}$ $(i \in[1 \ldots n], k \in[1 \ldots v])$ can be identified from the matrix $Q$ using Formula (6).

$$
W=\left(w_{i, k}\right)^{n \times v}=q_{i, k} \times F_{U}^{-1}\left(f_{k}\right), \forall u_{i} \in U
$$

In our example, the matrix $W$ is determined as follows, where $f_{1}$ and $f_{2}$ are the dominant features of $u_{1}$ and $u_{3}, f_{2}$ is the dominant feature of $u_{2}$ and $u_{5}$, and $f_{1}$ is the dominant feature of $u_{4}$ :

$$
W=\left(\begin{array}{ccccc}
f_{1} & f_{2} & f_{3} & f_{4} & \\
\mathbf{0 . 0 9 6 9} & \mathbf{0 . 0 9 6 9} & 0.0176 & 0.0088 & u_{1} \\
0.0969 & \mathbf{0 . 1 9 3 8} & 0.0176 & 0.0176 & u_{2} \\
\mathbf{0 . 0 9 6 9} & \mathbf{0 . 0 9 6 9} & 0.0176 & 0.0176 & u_{3} \\
\mathbf{0 . 1 9 3 8} & 0 & 0.0176 & 0.0088 & u_{4} \\
0 & \mathbf{0 . 1 9 3 8} & 0.0176 & 0.0176 & u_{5}
\end{array}\right.
$$

Step $\mathbf{5}$ - Specify a matrix representing the songs that have not been listened to by a user: We identify a matrix $\bar{M}$ with entries $\bar{m}_{j, k} \in Z^{+}(j \in[1 \ldots m], k \in[1 \ldots v])$ representing the songs that have not been listened to by user $u_{i}$. The matrix $\bar{M}$ can be constructed based on the matrices $N$ and $M$, where $\bar{M} \subset M$. For instance, $u_{1}$ has not listened to the songs $s_{2}$ and $s_{5}$. Thus, the matrix $\bar{M}$ of $u_{1}$ can be specified by getting the entries from $M$ that represent the latent features of $s_{2}$ and $s_{5}$.

$$
\bar{M}=\left(\begin{array}{cccc}
f_{1} & f_{2} & f_{3} & f_{4} \\
1 & 0 & 0 & 1 \\
0 & 1 & 1 & 1
\end{array}\right) s_{2}
$$

Step 6 - Predict the preferences of a user for unrated songs: A user's preferences for songs (that have not been listened to so far) can be determined based on the matrices $\bar{M}$ and $W$ and represented in a matrix $\bar{N}$ with entries $\overline{n_{i, j}} \in Z^{+}(i \in[1 \ldots n], j \in[1 \ldots m])$ :

$$
\bar{N}=W \times \bar{M} \text {. }
$$

Step 7 - Recommend to user $u_{i}$ the song with the highest predicted preference: Based on the matrix $\bar{N}$, the system recommends the songs with the maximum value of $\bar{n}_{i, j}(j \in[1 \ldots m])$ to user $u_{i}$. For instance, based on Formula (7), the matrix $\bar{N}$ of user $u_{1}$ can be specified as below, in which $s_{5}$ is selected as the recommended item:

$$
\bar{N}=\left(\begin{array}{ccc}
s_{1} & s_{5} & \\
0.1057 & \mathbf{0 . 1 2 3 3}
\end{array}\right) u_{1}
$$

The approaches presented in this subsection provide solutions for integrating emotions into recommender systems. However, existing solutions in most of the cases focus on the domains of

\footnotetext{
${ }^{6}$ For further details of this approach, we refer to Reference [2].
} 
movies and music. Emotion-related aspects receive less attention in other domains where emotions might have substantial impacts on the recommendation process. Examples thereof are tourism, retailing, and advertising. For instance, in the tourism domain, a user's emotions can affect his/her tourism attraction selection. The user chooses a museum to visit when he/she is feeling happy, but prefers going to the beach when he/she is feeling depressed. A real-time emotion analysis might be helpful for recommender systems to predict a user's current emotional state and then recommend more appropriate tourism attractions. Some approaches already try to incorporate emotions into tourism attraction recommendation (e.g., References [72, 83]). However, an in-depth integration of emotion-related aspects into tourism recommender systems is still an open issue.

\section{THE INFLUENCE OF GROUP DYNAMICS ON GROUP RECOMMENDER SYSTEMS}

Due to the importance in supporting group decision-making processes, group recommender systems have already triggered significant research efforts [51]. Compared to single-user recommender systems, the influence of psychological factors in group recommender systems should be considered differently. For instance, emotional factors in group decision scenarios have to be analyzed in the context of emotional collectives. For instance, family members are more likely to be affected emotionally compared to friends [168]. Group members' decision-making behavior could differ depending on the group type (e.g., homogeneous vs. heterogeneous groups). For instance, a user might be more caring about the preferences of family members than the preferences of colleagues. Consequently, the impact of group dynamics on the quality of group recommendations has to be investigated in more detail $[25,38,57]$. Supporting group decision making based on group recommendation techniques requires knowledge about group dynamics [20,37, 50]. In the following subsections, we discuss the impact of group dynamics on group decision making.

\subsection{Social Relationship}

Current research has pointed out that social relationships among group members significantly influence their decision-making behavior $[44,58]$. Users tend to rely more on recommendations from their friends or persons they trust than those generated by recommender systems [123]. This behavior can be applied in situations where group members change their item preferences to reach a consensus [44]. For instance, let us assume that a user does not like an item $i$. However, he/she might change his/her mind if he/she knows that this item is the favorite of his/her best friend. In this context, a social relationship can help to speed up the consensus achieving process. Currently, there exist various approaches to exploit social relationships in group recommendations. These are summarized in the following:

5.1.1 Trust Networks. Social relationships in a group can be represented in a trust network that helps to explore trust relationships among group members. Such a network can be used to predict group members' item ratings. An example of this approach is FilmTrust [60], which utilizes trust in social networks to predict movie ratings of a user. This approach achieves a better prediction quality than basic collaborative filtering. We now present an example to illustrate this approach.

Example 5. Given a group recommender system offering a social network component that enables users to maintain a list of friends, the rating prediction process can be conducted in the following steps:

Step 1 - Collect trust values: Trust values are collected in two ways: direct trust values or inferred trust values. For direct trust values, a user can directly provide a trust rating (e.g., through a rating scale of $[1 \ldots 10])$ to express how much he/she trusts his/her friend. For instance, Anna trusts Tom and gives him a trust value of 9. When giving a trust value, each user is advised to consider the following context: "If your friend would have consumed item $X$, how likely would you 
want to consume this item?" [60]. For inferred trust values, if a user has not specified the trust value for his/her friend, then the system can infer this value using a breadth first search-based algorithm [60]. The algorithm returns a trust value by finding paths from the user to his/her friend(s) and aggregating the trust values found along these paths.

Step 2 - Compute movie ratings: According to Step 1, the set of raters $R$ of a user $u$ is determined. Raters represent people whom user $u$ trusts. The recommended rating $r(u, m)$ of user $u$ for movie $m$ is the average of raters' movie ratings. These ratings are weighted by the trust value $t$ of user $u$ provided for each rater-see Formula (8). In this context, $t_{u \rightarrow v}$ is the trust value user $u$ gives to rater $v$ and $r_{v \rightarrow m}$ is the rating of rater $v$ for movie $m$.

$$
r(u, m)=\frac{\sum_{v \in R} t_{u \rightarrow v} \times r_{v \rightarrow m}}{\sum_{v \in R} t_{u \rightarrow v}}
$$

Assume Anna has two friends, Tom and Christina. She gives Tom and Christina the trust values of 8 and 4 , respectively $\left(t_{\text {Anna } \rightarrow \text { Tom }}=8\right.$ and $\left.t_{\text {Anna } \rightarrow \text { Christina }}=4\right)$. Tom rated the movie "Avengers" with 5 stars $\left(r_{T o m \rightarrow \text { Avenger }}=5\right)$, whereas Christina rated this movie with 3 stars $\left(r_{\text {Christina } \rightarrow \text { Avenger }}=3\right)$. The recommended rating $r$ (Anna, Avengers $)$ is calculated as follows:

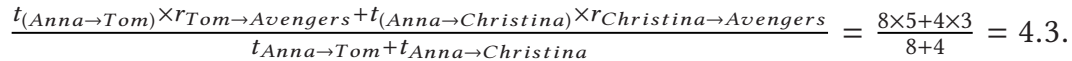

5.1.2 Social Networks. It is quite challenging to generate a trust network, since it requires explicit feedback from users, which is time-consuming and might lead to user fluctuation [123]. In this context, social-network based approaches were proposed to resolve the mentioned issue. These approaches enable the inference of trust knowledge from social networks such as Facebook or Twitter [35, 59, 123]. Social networks contain user information (e.g., personal information, interests, pictures) that can be extracted to estimate the trust level between two users [123]. This way, users are not required to provide explicit trust-related information. Existing studies using these approaches are the following:

Christensen and Schiaffino [35] introduced a group recommender system that takes into account social factors when determining group recommendations. Examples of such factors are trust relationship, social similarity, and social centrality. Trust relationship indicates the degree of cohesion between group members. Social similarity reflects characteristics shared among group members (e.g., shared activities, friends, and interests). Social centrality refers to group members' reputation in the social network. In this study, the authors generated group recommendations based on a group model constructed by aggregating individuals' models. The authors took into account two important factors: (1) "which items (of individuals' models) to be included in the group model?" and (2) "how to aggregate individuals' preferences to obtain a collective preference?". To identify group recommendations, the authors proposed a hybrid approach that combines collaborative filtering and content-based filtering. This approach detects implicit similarities between the ratings of group members. To aggregate individual preferences, the authors considered the mentioned social factors. These help to identify the degree of influence among group members and to provide possible preference changes. The idea is that the closer the relationship between group members, the higher the influence on each other's opinions. In other words, in "close-relationship groups", there is a higher probability that group members are willing to adapt their preferences for the sake of consensus.

In the same line of research, Delic and Masthoff [37] analyzed the impact of social closeness and social centrality on group decision outcomes. This approach was guided by the concept of "prominent actor." A prominent actor in a social network is "the one who is the object of extensive ties, focusing on the actor as a recipient" [169]. Based on this concept, the authors examined if socially central members of a group report higher group identity and have their individual preferences 
closer to the final group choice. Besides, the authors took into account the relationship between group identity and group decision making. Group identity is defined as "the individuals' self-concept derived from their knowledge of their membership to a social group together with the value and emotional significance attached to that membership" [149]. The experimental results show that socially central members of the group tend to be perceived as more influential in the group decision-making process. Besides, social relationships help to reach the level of group identity, where members are aware of the feeling of each other. The social closeness of a group is related to the perceived group similarity (in terms of preferences). The authors pointed out that socially central group members are significantly happier with the final group choice, even if they are the "disadvantaged" in the decision-making process.

In another study, Quijano-Sanchez et al. [123] modeled user profiles based on different factors extracted from Facebook and then computed the trust between two users $u$ and $v$ in a given group. The discovered factors describe the role of users in a social network:

$-f_{1}(u, v)$ : Distance in the social network, which checks if two users are friends.

$-f_{2}(u, v)$ : Number of mutual friends.

$-f_{3}(u, v)$ : Intensity of the relationship, which indicates how often a user appears on the wall of his/her friends.

$-f_{4}(u, v)$ : Intimacy of the relationship, which can be classified based on keywords mentioned in their wall interactions.

$-f_{5}(u, v)$ : Duration, which measures how long users know each other.

$-f_{6}(u, v)$ : Reciprocal services, which determines the number of videos/songs/webs that users have posted on each others' walls.

$-f_{7}(u, v)$ : Structural variable, which counts common interests described in the users' profiles and how many groups they have joined.

$-f_{8}(u, v)$ : Social distance, which shows how users share information in their profiles (e.g., political beliefs, school/universities, religious beliefs, and demographics situation).

$-f_{9}(u, v)$ : Status, which represents the relationship between two users.

$-f_{10}(u, v)$ : Pictures, which shows the percentage of pictures in which users appear together.

Thereafter, the authors estimated the mentioned factors by analyzing the profiles of the users participating in their experiment. Based on the identified factor values, the social trust level between users $u$ and $v$ can be calculated by combining the mentioned factors. Since these factors may have different impacts on the recommendation process, the trust between two users is a weighted average of the factors (see Formula (9)), where $w_{i} \in[0 \ldots 1]$ indicates the impact level of factor $f_{i}$ $\left(\sum_{i=1}^{10} w_{i}=1\right)^{7}$

$$
\operatorname{trust}(u, v)=\sum_{i=1}^{10} w_{i} \times f_{i}(u, v)
$$

For illustration purposes, we now present an example of how to calculate the social trust level between two users based on the approach of Quijano-Sanchez et al. [123].

Example 6. Assume Tina and Bob are in a group of friends. Their relationship is described by the 10 factors mentioned above. The values and corresponding weights of these factors are summarized in Table 10. In this example, the factors mutual friends $\left(f_{2}\right)$, duration $\left(f_{5}\right)$, structural variable $\left(f_{7}\right)$, status $\left(f_{9}\right)$, and pictures $\left(f_{10}\right)$ have significant impacts on social trust (weight $\left.>0\right)$. Based on Formula (9), the social trust level between Tina and Bob is calculated as follows:

$$
\operatorname{trust}(\text { Tina }, B o b)=(0.43 \times 0.5)+(0.17 \times 0.5)+(0.14 \times 0.45)+(0.07 \times 0.3)+(0.2 \times 0.19)=0.422
$$

\footnotetext{
${ }^{7}$ The weight (the impact level) $w_{i}$ of factor $f_{i}$ was measured using an experimental approach. For further details of this approach, we refer to Reference [123].
} 
Table 10. Example 6 - Factors Describing the Relationship between Two Users

\begin{tabular}{|c|c|c|c|}
\hline Factor & Description & Value & Weight \\
\hline$f_{1}$ & They are direct friends & 1.0 & 0 \\
\hline$f_{2}$ & They have 12 mutual friends & $\mathbf{0 . 5}$ & $\mathbf{0 . 4 3}$ \\
\hline$f_{3}$ & Every month, they write each other on their walls & 0.3 & 0 \\
\hline$f_{4}$ & Friendly & 0.5 & 0 \\
\hline$f_{5}$ & They have known each other for more than 3 years & $\mathbf{0 . 5}$ & $\mathbf{0 . 1 7}$ \\
\hline$f_{6}$ & So far, they have shared 2 games on Facebook & 0.3 & 0 \\
\hline$f_{7}$ & Both of them like watching movies and reading books & $\mathbf{0 . 4 5}$ & $\mathbf{0 . 1 4}$ \\
\hline$f_{8}$ & They share three properties: educational, religious, and demographics information & 0.7 & 0 \\
\hline$f_{9}$ & Their current status is friends & $\mathbf{0 . 3}$ & $\mathbf{0 . 0 7}$ \\
\hline$f_{10}$ & There are 20 pictures where they appear together & $\mathbf{0 . 2}$ & $\mathbf{0 . 1 9}$ \\
\hline
\end{tabular}

The value and weight of each factor are estimated using the approach proposed in Reference [123]. In this example, only five factors $f_{2}, f_{5}, f_{7}, f_{9}$, and $f_{10}$ have significant impacts on social trust (weight $>0$ ).

Social-network-based approaches have brought significant improvements in terms of recommendation accuracy [44]. However, these approaches have been only tested in specific domains (e.g., movies [35, 123] and tourism [37]), which raises the question of generalizability. These approaches need to be further evaluated in other domains to prove their applicability. Following these approaches, every user who participates in a group decision must belong to a social network. Due to the popularity of social networks and the tendency of using such networks to organize real-life events, these approaches could also be easily integrated into the discussed group recommendation scenarios [123].

\subsection{Emotional Contagion}

In Section 4, emotions have been analyzed in the context of single-user oriented recommendation. In group recommendation scenarios that focus on the interaction among group members, emotions are taken into account in the concept of emotional contagion. In this context, the emotional state of a user is unintentionally and uncontrollably influenced by the emotional states of others [50,141]. In group settings, group members' emotions can affect the emotions, thoughts, and behaviors of others [65]. For instance, in a software development team, the mood of a team leader can be transferred to stakeholders and thereby impacts the effort and coordination of the whole team. This phenomenon can also be transferred " $a s$-is," for instance, when watching a movie with friends, a group member might not enjoy the movie if he/she knows that his/her best friend does not like the movie [101]. The existence of emotional contagion may depend on users' personality. For instance, selfish users are usually not affected by others, whereas tolerant users tend to be easily influenced. Furthermore, relationships between group members play a role; for instance, users are more likely to be affected by whom they love [101].

Emotional contagion has been investigated recently in sequence recommendations (e.g., recommending a sequence of TV programs to family members). Masthoff and Gatt [102] confirmed that the emotional state of a group member could be affected by other group members. Chen and $\mathrm{Pu}$ [34] proposed a group music recommender system so-called GroupFun where an emotion annotation tool called $\mathrm{CoFeel}$ is used to annotate and visualize the emotional states of group members. The authors investigated the emotional feedback of users regarding recommendations. The experimental results show that displaying each other's emotions with regard to recommended items positively influences the outcome of a group decision process. Being aware of the emotional states of other group members helps to better negotiate group-related issues (e.g., resolving conflicts and achieving consensus) [33]. 


\subsection{Group Personality Composition}

Group recommender systems try to take into account the preferences of individual group members and attempt to satisfy each group member [122]. In heterogeneous groups, a conflict situation may arise more easily if the preferences of group members are incompatible [127]. Besides, it is shown that the overall satisfaction of a group cannot be always achieved by aggregating group members' preferences [122]. Thus, a novel method for group recommendation needs to be developed, in which different types of individual personalities are considered.

Rossi and Cervone [129] proposed an approach that takes into account the agreeableness personality trait (see Section 3.1) in the group recommendation context. The authors argued that agreeable people tend to compromise and care about the entire group's satisfaction. Based on this idea, the authors proposed a solution relying on the definition of a utility function that models the altruistic behavior of group members. The utility function evaluates user ratings for items, considering the preferences of the whole group and the preferences of agreeable users. The authors used a model developed by Charness and Rabin [29] to define the underlying utility function ${ }^{8}$ that maximizes social welfare. This value measures how much a person likes to increase social surplus, caring especially about helping him/herself and others with low payoffs [29].

Nguyen et al. [109], Quijano Sanchez et al. [122], and Recio-Garcia et al. [127] proposed approaches that maximize group satisfaction by considering the personality of group members. In these studies, the authors characterized users using the Thomas-Kilmann Conflict Mode Instrument (TKI) model [156] that describes a user's behavior in conflict situations according to two dimensions: assertiveness and cooperativeness [127]. The combination of these dimensions results in different personality modes: competing, collaborating, avoiding, accommodating, and compromising (see also Subsection 3.1). Although these studies share the idea of exploring the behavior of individual group members for conflict resolution, they show some differences in the modeling of assertiveness and cooperativeness. Nguyen et al. [109] modeled assertiveness based on the probability that group members propose items with a high personal utility. The higher the probability, the higher the assertiveness. In contrast, cooperativeness is modeled based on the probability of a user to give positive and negative evaluations to items proposed by other group members. A cooperative person has a high probability of giving positive feedback and a low probability of giving negative feedback. Quijano Sanchez et al. [122] and Recio-Garcia et al. [127] estimated the assertiveness and cooperativeness of a user based on the sum of the coefficients of his/her personality modes. These dimensions are combined to estimate Conflict Mode Weight (CMW), representing how selfish or cooperative a group member is. Thereafter, the rating of a group member $u$ for a specific item can be predicted by considering the personality difference between user $u\left(C M W_{u}\right)$ and another user $v\left(C M W_{v}\right)$ in the group [123].

In this subsection, we present an algorithm proposed by Recio-Garcia et al. [127], which generates group recommendations by aggregating the recommendations of individual group members (i.e., aggregated predictions [47]). The $C M W s$ are included in the aggregation process. We now present an example to illustrate this algorithm.

Example 7. Given a group of three users $G=\left\{u_{1}, u_{2}, u_{3}\right\}$ and a list of four items $I=\left\{i_{1}, i_{2}, i_{3}, i_{4}\right\}$, the rating predictions for each group member $u$ and each item $i$ are shown in Table 11.

Assertiveness and cooperativeness can be either categorized as high or low (see Table 12). ${ }^{9}$ These dimensions are evaluated by summing the coefficients of all personality modes extracted from Table 13. The TKI test determines the degree of cooperativeness and assertiveness for each user.

\footnotetext{
${ }^{8}$ For further details of the utility function, we refer to Reference [129].

${ }^{9}$ For the determination of the high/low categories of the users regarding TKI mode, we refer to Reference [85].
} 
Table 11. Example 7 - Predicted Ratings of Group Members $u$ for Items $i$

\begin{tabular}{|l|l|l|l|l|}
\hline & $i_{1}$ & $i_{2}$ & $i_{3}$ & $i_{4}$ \\
\hline$u_{1}$ & 1 & 3 & 4 & 2 \\
\hline$u_{2}$ & 2 & 5 & 3 & 1 \\
\hline$u_{3}$ & 3 & 2 & 1 & 4 \\
\hline
\end{tabular}

Table 12. Example 7 - Assertiveness and Cooperativeness Degrees for Group Members Described by the Personality Modes Competing, Collaborating, Compromising, Avoiding, and Accommodating [85]

\begin{tabular}{|c|c|c|c|c|c|}
\hline user & competing & collaborating & compromising & avoiding & accommodating \\
\hline$u_{1}$ & high & high & low & low & low \\
\hline$u_{2}$ & high & low & low & low & low \\
\hline$u_{3}$ & low & high & high & low & high \\
\hline
\end{tabular}

Table 13. Example 7 - Coefficients for Determining Assertiveness and Cooperativeness [127]

\begin{tabular}{|c|c|c|c|c|}
\hline \multirow{2}{*}{ TKI mode } & \multicolumn{2}{|c|}{ assertiveness } & \multicolumn{2}{c|}{ cooperativeness } \\
\cline { 2 - 5 } & high & low & high & low \\
\hline competing & 0.375 & -0.075 & -0.15 & 0 \\
\hline collaborating & 0.375 & -0.075 & 0.375 & -0.075 \\
\hline compromising & 0 & 0 & 0 & 0 \\
\hline avoiding & -0.375 & 0.075 & -0.375 & 0.075 \\
\hline accommodating & -0.15 & 0 & 0.375 & -0.075 \\
\hline
\end{tabular}

Table 14. Example 7 - Assertiveness, Cooperativeness, and CMW Estimates of Group Members

\begin{tabular}{|c|c|c|c|}
\hline user & assertiveness & cooperativeness & CMW \\
\hline$u_{1}$ & $0.375+0.375+0+0.075+0=0.825$ & $-0.15+0.375+0+0.075-0.075=0.225$ & 0.8 \\
\hline$u_{2}$ & $0.375-0.075+0+0.075+0=0.375$ & $-0.15-0.075+0+0.075-0.075=-0.225$ & 0.8 \\
\hline$u_{3}$ & $-0.075+0.375+0+0.075-0.15=0.225$ & $0+0.375+0+0.075+0.375=0.825$ & 0.2 \\
\hline
\end{tabular}

The information in Tables 12 and 13 is combined for assertiveness and cooperativeness estimates, which are summarized in Table 14.

Based on the assertiveness and cooperativeness estimates, the Conflict Mode Weight (CMW) of each user is calculated using Formula (10). The CMW value stays in the range of $[0,1]$, where 0 reflects a very cooperative person and 1 reflects a very selfish one (see the last column of Table 14).

$$
\operatorname{CMW}(u)=\frac{1+\operatorname{assertiveness}(u)-\text { cooperativeness }(u)}{2}
$$

To find a list of recommended items for the group, the recommendation algorithm performs the following steps: 
Step 1: Recommend $k$ items that are the best for each group member $u\left(B e s t_{u}\right)$ using, for instance, a collaborative filtering approach [77]. We assume the recommendation list $(k=2)$ given to each group member as follows: Best $t_{u_{1}}=\left\{i_{2}, i_{3}\right\}$, Best $t_{u_{2}}=\left\{i_{2}, i_{3}\right\}$, Best $t_{u_{3}}=\left\{i_{1}, i_{4}\right\}$.

Step 2: For each item $i$ in Best $t_{u}$, execute the CMW merging function (MinBest $u_{u i}$ ) based on the minimization misery procedure [111], which merges the preferences of group members using a social choice function so-called Least Misery [47]. With this function, a group's happiness is the minimum of the individual members' happiness scores [111]. MinBest $t_{u i}$ is implemented using Formulae (11) and (12), where $r_{v i}$ is the rating of user $v$ for item $i$ and the $\alpha$ value has been experimentally computed to modify the impact of the CMW differences (in this example, we assume $\alpha=1$ ). The MinBest ${ }_{u i}$ value reflects that fact that: "If user $v$ is more assertive than user $u\left(C M W_{u v}<0\right)$, then his/her rating will downgrade the MinBest ${ }_{u i}$." This way, the best items from $u$ could have a lower misery rating than those from $v$. Hence, the favorite items of $v$ have more chances to be recommended.

$$
\begin{aligned}
& \text { MinBest }_{u i}=\min _{v}\left(r_{v i}+C M W_{u v}\right) \\
& C M W_{u v}=\left(C M W_{u}-C M W_{v}\right) \times \alpha
\end{aligned}
$$

Following Formulae (11) and (12), the merging function MinBest $t_{i u}$ of a group member $u$ for each item $i$ in Best ${ }_{u}$ is calculated as follows:

$$
\begin{aligned}
& \text { MinBest } t_{u_{1} i_{2}}=2.6 \sqrt{ }, \text { MinBest } \\
& \text { MinBest }_{u_{1} i_{3}}=1.6 \\
& \text { MinBest }_{u_{3} i_{1}}=2.6 \sqrt{ }, \text { MinBest } \\
& u_{u_{2} i_{3}}=1.6, \text { MinBest } \\
& u_{u_{3} i_{4}}=2.2 .
\end{aligned}
$$

Step 3: Select $N$ items fulfilling a selection criterion. In this algorithm, items with the highest MinBest value are selected for the group recommendation. Assume $N=1, i_{2}$ is chosen as the item recommended to the group.

\subsection{Conformity}

In group settings, conformity indicates a process where the opinion of a group member is influenced by the opinion of others [50,101]. There are two types of conformity: normative influence and informational influence. The former indicates situations where a group member wants to be a part of the group and expresses his/her opinion like the rest of the group, even though he/she still has a different opinion. The latter refers to situations where a group member changes his/her own opinion, since he/she believes in the group's opinion [101]. Besides, based on empirical results, Nguyen and Ricci [108] investigated three conformity types: (1) independence - the group member does not change his/her preference; (2) conversion - the group member's preference tends to become similar to other group members' preferences; and (3) anti-conformity - the group member's preference becomes more divergent. In group recommender systems, conformity can be used to better predict the preparedness level of individual group members to adapt their initial evaluations for items [50]. The existing literature shows different approaches to estimate the conformity level of a group member. For instance, Masthoff and Gatt [102] proposed an approach to calculate the conformity level of a group, which is composed of multiple subgroups with different opinions. The conformity level of a group is the sum of subgroups' information influence values. The information influence value of a specific subgroup is estimated based on the following three factors: the influence factor (defined by Latané and Wolf [91]), the difference between group members' opinions, and the number of group members outside that subgroup. Alternatively, Quintarelli et al. [125] presented a model to measure the influence of a group member based on the frequency of his/her preference appearing in the final group choice (i.e., the higher the frequency, the higher the influence of a group member). Although conformity plays a major role in leading groups to consensus-based decisions, too much conformity can result in groupthink. Groupthink is a bias causing negative 
consequences for group productivity, since the individual creative and intellectual contributions of group members tend to be suppressed [164] (see also Section 6.5). Hence, conformity in group decision making has to be considered in more detail.

\subsection{The Role of Group Members}

Group recommendations are often generated by merging individual group members' preferences into a group profile, which represents the preferences of the whole group [47]. When aggregating the data of individual group members, it is natural to allow some users to have a stronger influence than others (i.e., group members with a more important role will have a higher weight than others). The influence of group members' role on group decision-making processes has been investigated in many studies regarding family decisions. The early literature assumes that wives usually play the most important role in purchasing situations [138]. Later research breaks down family decisions into sub-decisions and shows that group decisions are usually shared between husbands and wives with different levels of responsibility depending on the nature of sub-decisions [158]. Husbands are more dominating in sub-decisions on the length of trips, timing of vacations, and expenditure, whereas sub-decisions regarding taking care of children, transportation modes, activities, and destinations are all joint husband-wife choices [78]. The role of children has been investigated in some studies. For instance, Thornton et al. [158] analyzed group members' needs in two- or more-generation families. They found out that, in holiday decisions, children have a significantly higher impact on group decisions: on the one hand, through their physical needs (e.g., meal-time arrangement or sleeping need); on the other hand, through their ability to negotiate with parents.

In this line of research, several studies have been conducted to deal with role-based models, which weight members according to their role in the group [7, 10, 15, 39, 58]. For instance, Ardissono et al. [7] distinguished different user types (e.g., children and adults with and without disability) and assign higher weights for vulnerable users (e.g., children and the disabled). Atas et al. [10], and Gartrell [58] proposed approaches to estimate item ratings that emphasize the role of group members with a high expertise level. For instance, in a software development project, project managers and domain experts have a higher impact on group decisions compared to other stakeholders. Berkovsky et al. [15] proposed a role-based model that weights users according to their responsibility. For instance, in a family party, a group member who organizes the party should have a higher weight/importance than other group members who are just participants. Delic et al. [39] defined the role of a group member based on his/her "preference centrality" in the group. The preference centrality of a member is determined by the level of information and knowledge that he/she shares with the rest of the group [82]. Preference centrality is extracted from the group preference network and used in a weighted aggregation strategy to generate group recommendations.

\subsection{Fairness}

In group recommender systems, fairness refers to a state where group members' preferences need to be considered as far as possible when making group recommendations. Taking into account fairness has a positive effect on a group recommendation process, since higher satisfaction of individual group members can be achieved [165]. Previous research on group recommender systems neglected this aspect. More recent studies have attempted to explore possibilities of increasing the fairness perception of users in group decision making [172]. These studies take into account different fairness aspects; for instance, Carvalho and Macedo [26] considered a group recommendation task from the perspective of game theory [173], in which a group recommendation is fair if it balances between satisfying a group member's interest and avoiding the dissatisfaction of others. Serbos et al. [136] took into account fairness aspects in group decisions based on the fair division 
theory in Economics [19], which divides resources to groups of users in such a way that everyone is happy.

In this subsection, we focus on fairness aspects from the user perspective, i.e., how group members perceive fairness in group decision making and how fairness perception changes group members' decision -making behavior. The fairness perception of group members can differ depending on the decision type (e.g., repeated or non-repeated decisions) [49]. In non-repeated (or seldom-repeated) decisions (e.g., choosing a new house to buy for the whole family), group members might expect that fairness should be considered right in the ongoing decision (i.e., no one's preferences should be ignored). In repeated decisions periodically taken by the same group (e.g., choosing a movie to watch every weekend), fairness should be taken into account in previous and future decisions. Group members should be aware that users whose preferences were not considered in the past will have a higher weight in ongoing or future decisions. Similar scenarios can be found in software engineering, where stakeholders have to make decisions on release plans every couple of weeks to decide which requirements should be implemented next. Each requirement is evaluated according to three dimensions: risk, profit, and effort [10]. The importance of each dimension can be perceived differently by stakeholders (see Table 15). It might be the case that the preferences of some stakeholders are more often considered than the preferences of others. To foster fairness within a group, the preference weights of stakeholders should be adapted [145] (see Formulae (13) and (14)). Stakeholders whose preferences have not been considered in previous decisions should have a higher weight in upcoming decisions [165]. Formula (13) estimates the perceived fairness of each user $u_{i}$ (in group $G$ ) in terms of the share of the number of supported preferences in relation to the number of group decisions. The lower the value, the lower the degree of fairness experienced by user $u_{i}$. Formula (14) represents an approach to increase fairness in upcoming recommendation sessions. If the fairness in previous sessions was low, then a corresponding upgrade of user-specific importance weights is triggered for each dimension (i.e., risk, profit, and effort).

$$
\begin{gathered}
\text { fair }\left(u_{i}\right)=\frac{\# \text { supportedpreferences }\left(u_{i}\right)}{\# \text { groupdecisions }} \\
w^{\prime}\left(u_{i}, \operatorname{dim}_{j}\right)=w\left(u_{i}, \operatorname{dim}_{j}\right) \times\left(1+\left(\frac{\sum_{u \in G} \text { fair }(u)}{|G|}-\operatorname{fair}\left(u_{i}\right)\right)\right)
\end{gathered}
$$

In the following, we present an example (in software engineering context) showing how fairness can be taken into account in repeated decisions.

Example 8. Assume three stakeholders $\left(s_{1}, s_{2}\right.$, and $\left.s_{3}\right)$ have to jointly make decisions on release plans every two months. In each decision, the stakeholders evaluate requirements according to the three dimensions risk, profit, and effort. The determined dimension-specific stakeholder weights are shown in columns 2-4 (Table 15). Up to now, the stakeholders made five decisions, and the estimated perceived fairness of each stakeholder is summarized in column 5 (Table 15). Using Formula (14), the estimated fairness levels (the adapted weight) of stakeholders are shown in columns 6-8 (Table 15). The weights of stakeholder $s_{3}$ for all dimensions have been increased, since he/she has the lowest estimated perceived fairness level (there were three out of five decisions where his/her preferences were not considered). In contrast, the weight of stakeholder $s_{1}$ has been decreased, since his/her preferences have been favored in all previous decisions.

Fairness aspects can also be taken into account depending on the underlying item domain. One finding from Felfernig et al. [49] indicates that the preferred preference aggregation strategies in high-involvement item domains (domains with high related decision efforts, e.g., decisions on tourism packages or financial services) differ from those in low-involvement item domains (domains with low related decision effort, e.g., decisions on music or movies). In the former domains, groups 
Table 15. Example 8 - An Example of the Adaptation of Stakeholder Weights for Dimensions in the Context of Requirements Prioritization

\begin{tabular}{|c|c|c|c|c|c|c|c|}
\hline \multirow{2}{*}{ Stakeholders } & \multicolumn{3}{|c|}{ Weight $(w)$} & \multirow{2}{*}{ Fairness (fair) } & \multicolumn{3}{|c|}{ Adapted weight $(w)$} \\
\cline { 2 - 4 } \cline { 6 - 8 } & risk & profit & effort & & risk & profit & effort \\
\hline \hline$s_{1}$ & 0.5 & 0.8 & 0.7 & $5 / 5=1$ & 0.33 & 0.53 & 0.47 \\
\hline$s_{2}$ & 0.6 & 0.7 & 0.8 & $3 / 5=0.6$ & 0.64 & 0.75 & 0.85 \\
\hline$s_{3}$ & 0.4 & 0.6 & 0.6 & $2 / 5=0.4$ & 0.51 & 0.76 & 0.76 \\
\hline
\end{tabular}

Each requirement is evaluated according to three dimensions: risk, profit, and effort [10]. The weights $(w)$ of stakeholder $s_{3}$ for all dimensions have been increased, whereas those of stakeholder $s_{2}$ almost stay the same, and those of stakeholder $s_{1}$ have been decreased (since the preferences of $s_{1}$ have been favored in previous decisions).

prefer using the Least Misery strategy, which minimizes the misery of group members, whereas they tend to apply Average Voting in the latter domains.

\subsection{Consensus}

In heterogeneous groups where group members can have different expectations regarding recommendation options, finding a recommendation that satisfies each group member becomes difficult. Conflicts between group members might arise when their item preferences are incompatible. Therefore, a consensus -making process is required to resolve such conflicts and help group members to agree on a solution. This process also ensures a high satisfaction of group members with the recommended items [165]. One of the greatest concerns is how to achieve consensus or how to deal with diverging preferences among group members. Studies in References $[28,113,116]$ show that consensus can be obtained through a negotiation process, which ends when the consensus level reaches a pre-defined threshold. Consensus can also be obtained by pointing out disagreements between the preferences of group members [6].

Another approach to achieve consensus is to utilize the role of group leaders to foster preference harmonization [167]. This approach applies a centrality consensus model, in which a user-who is an expert in the domain-is selected as the group leader and named as Supra Decision Maker (SDM). The SDM plays the role of a central advisor to measure the preference similarity between users. The consensus degree on an item is calculated based on the preference similarity between the $S D M$ and each group member. A threshold is pre-defined by the group and compared with the similarity scores to determine if a group member has achieved a consensus with the SDM on a specific item. If not, then the group member has to adapt his/her preferences. Thereafter, the similarity calculation process is repeated. The consensus-achieving process terminates when a specific number of items (e.g., more than half of the items [167]) achieve a consensus.

Finally, consensus achieving in group decision making can be supported by analyzing the history of group decisions. Tran et al. [165] took into account the satisfaction of group members from previous decisions as a criterion to convince group members to agree on an item. For instance, to help group members achieve a consensus on item $X$, an explanation can be formulated as follows: "Item $X$ has been chosen for the group, since it supports the preferences of user A who was treated less favorably in the last three decisions." This explanation helps to increase the consensus perception of group members.

\section{DECISION BIASES IN RECOMMENDER SYSTEMS}

In addition to the aspects of personality, emotions, and social factors as mentioned in the previous sections, decision making can be affected by decision biases, which could lead to sub-optimal decision outcomes. In fact, while interacting with recommender systems, in most cases, users do 
Table 16. An Example Asymmetric Dominance Decoy Effect in Which Laptop 1 Is a Competitor Item (C), Laptop 3 Is a Target Item

$(T)$, and Laptop 2 Is a Decoy Item $(D)$

\begin{tabular}{|c|c|c|c|}
\hline & Laptop 1 (C) & Laptop 2 (D) & Laptop 3 (T) \\
\hline Processor & $2.0 \mathrm{GHz}$ & $2.0 \mathrm{GHz}$ & $2.9 \mathrm{GHz}$ \\
\hline Storage & $256 \mathrm{~GB}$ & $256 \mathrm{~GB}$ & $512 \mathrm{~GB}$ \\
\hline Price & $€ 799$ & $€ 899$ & $€ 859$ \\
\hline
\end{tabular}

In this example, item $T$ dominates item $D$ in all dimensions. This is not the case with item $C$. Therefore, $T$ appears to be the better item compared to $C$.

not have a clear picture of their preferences in mind before starting a decision process [30]. In this context, instead of optimizing decisions, users are more likely to apply decision heuristics, which can result in decision biases. There exist plenty of decision biases that could influence the decision-making behavior of users. However, in this article, we only focus on decision biases that have been studied in the context of recommender systems, for both single-user and group recommendation scenarios. ${ }^{10}$ In this context, we focus on decoy effects, serial position effects, anchoring effects, framing effects, and group think. For each bias, we first explain the bias and then discuss its relevance in the recommendation context.

\subsection{Decoy Effects}

Decoy effects are cognitive phenomena usually detected in single-user recommender systems. Due to these effects, users tend to change their selection behavior regarding a target item $T$ and a competitor item $C$ when being confronted with a decoy item $D$ [154]. More precisely, the inclusion of decoy items in the recommended item list can increase the selection probability of target items. In this context, a decoy item is an alternative that is in one way or another inferior to all other alternatives [98]. Table 16 depicts an example of asymmetric dominance, which is a specific type of decoy effects where the target item dominates the decoy in all dimensions.

Application of decoy effects: Decoy effects can be exploited in recommender systems for the following purposes:

-Increasing the selection probability of target items: It has been shown in various domains (e.g., financial services [153], hotel rooms [151], and game characters [152]) that decoy effects can help to increase the attraction of target items with respect to competitor items. However, the exploitation of these effects in recommender systems comes along with ethical issues, since companies might utilize decoy items just for selling their products rather than offering optimal products to customers [98].

-De-biasing decoy effects and generate explanations for recommendations: Knowledge about decoy items can be exploited for de-biasing purposes. Felfernig et al. [53] identified dominance relationships among different items in a candidate set and then eliminated decoy items from the result set. Moreover, decoy items can also help to generate adequate explanations. For instance, "item $T$ is the clear winner, since it dominates item $D$ in both dimensions (processor and storage), and the price is just a bit higher" [46]. In recommender systems, such an explanation helps to explain why item $T$ is a good option. Also, it increases a user's trust in the recommender system and motivates him/her to buy the suggested item [31].

- Making a decision more efficiently: Decoy items help users to resolve cognitive dilemmas in situations when they are unsure about which item to choose. This way, decoy items can increase users' decision confidence and accelerate the decision-making process [46, 98].

\footnotetext{
${ }^{10}$ For further details on decision biases, we refer to References $[46,48,126]$
} 


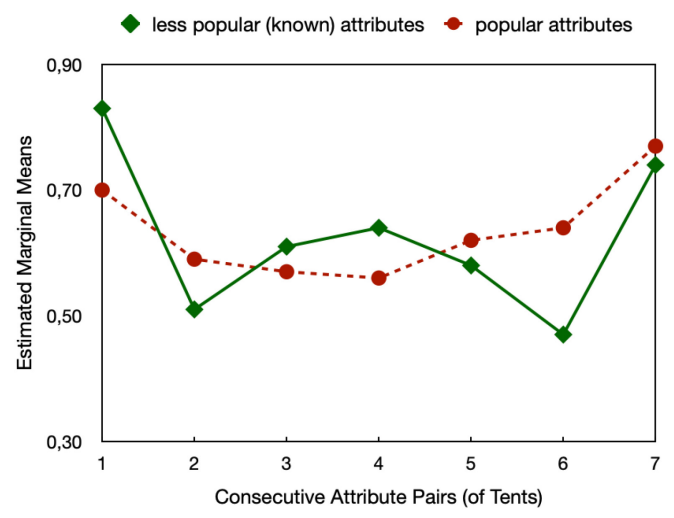

Fig. 4. An example of serial position effects where item attributes are presented in a sequence [52]. Item attributes located at the beginning and at the end of a sequence are more likely to be recalled than those in the middle, even if the attributes at the beginning and at the end are the less popular ones.

\subsection{Serial Position Effects}

Serial position effects (also known as primary/recency effects) are decision biases triggered when items are presented in the form of a list $[98,154]$. These biases usually occur in single-user recommendation scenarios, where users tend to focus on evaluating items shown at the beginning and at the end of a list. Felfernig et al. [46,52] showed that items at these two positions are more likely to be evaluated than others (see Figure 4). Serial position effects can change the selection behavior of users when interacting with recommender systems. For instance, in personnel decision making, Highhouse and Gallo [66] found out that candidates interviewed at the end of a recruitment process have a higher probability of being selected. Stettinger et al. [147] investigated serial position effects in the restaurant domain where restaurant reviews of users are analyzed. The authors show that different arrangements of the same arguments can lead to significantly different perception levels of users concerning restaurant attractiveness.

Serial position effects also affect recommendation scenarios for groups. Tran et al. [166] investigated the influence of these effects when the same group of users has to continuously make a sequence of decisions in different item domains (e.g., low-involvement and high-involvement item domains). The authors analyzed if the order of decision tasks causes different decision-making strategies of group members. The experimental results show that group members' decision strategy for high-involvement items are kept, i.e., are re-used in follow-up low-involvement item decisions (but not vice versa).

Application of serial position effects: Serial position effects can be exploited in e-learning recommender systems. These effects can increase the frequency of interacting with questions in e-learning systems (for single-user recommendation scenarios). In online courses, e-learning systems are used to support learners' training processes. Some systems allow learners to proceed many training rounds on the same topic with an attempt to answer all the questions correctly [148]. In each training round, a list of questions should be recommended to the learner. The recommendation list can be generated based on the learner's training performance or the questions' difficulty level. For instance, difficult questions answered wrongly by the learner in the previous training rounds should be recommended to him/her in the next training rounds. In this context, one potential solution for applying serial position effects is to place the most relevant questions at the beginning or at the end of the recommendation list. This way, these questions have a high probability of being accessed by the learner. 


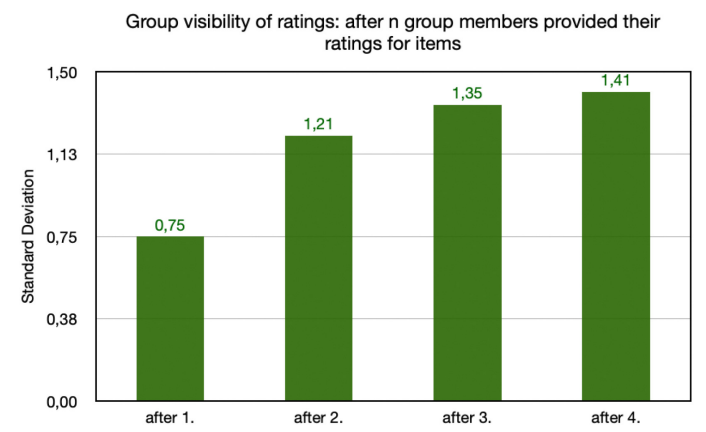

Fig. 5. Anchoring effects in requirements engineering [146]. The earlier the individual preferences are disclosed to other group members, the lower the group members' rating difference. For instance, if the ratings of group members are shown after one user has articulated his/her preferences, then the group rating difference is 0.75 , whereas after four users, the difference is two times higher (1.41).

\subsection{Anchoring Effects}

Anchoring effects are cognitive biases where users often heavily rely on the first information (an anchor) when evaluating decision alternatives [46]. These effects occur, for example, in single-user recommender systems, where predictions generated by recommender systems impact user preferences at the time of consumption. Adomavicius et al. [4] conducted several surveys in different item domains (e.g., TV shows, jokes, and songs) and found out that a recommendation provided by an online system serves as an anchor when consumers form their preferences. The authors detected that user preferences appear malleable and can be significantly influenced by recommendations.

Anchoring effects also occur in group recommendation scenarios. In group recommender systems, these biases can be triggered when one group member's evaluations for items are influenced by others whose preferences were articulated earlier. Social-psychological studies confirm that the disclosure of group members' preferences in the early phase of the group decision-making process can result in a confirmation bias, in which group members tend to focus on discussing available information rather than exploring and sharing new decision-relevant information [48]. Stettinger et al. [146] analyzed the impact of anchoring effects in requirements engineering scenarios where groups of stakeholders have to decide on which requirements should be implemented in their software project. The authors claimed that the occurrence probability of an anchoring effect increases if individual group members' preferences are disclosed to others in the early phase of a group decision-making process (see Figure 5).

Application of anchoring effects: Anchoring effects can be exploited in recommender systems for the following purposes:

-Increasing the willingness of users to pay for products: Recent research has shown that online recommendations displayed to users can significantly increase their willingness to purchase items in the line of the recommendations $[4,86]$. However, similar to decoy effects, ethical aspects have to be kept in mind when exploiting anchoring effects in recommender systems.

-De-biasing anchoring effects: Being aware of these effects is critical to find a way of counteracting. For instance, in single-user recommender systems, since system-predicted ratings can distort users' preferences, Adomavicius et al. [3] proposed and investigated two possible approaches to de-bias the preference ratings of a user: post hoc rating adjustment and bias-aware interface design for rating collection. The first approach follows the idea of adjusting user-submitted ratings. The second approach tries to prevent anchoring effects by utilizing specific user interfaces for rating collection. In this context, the authors investigated various rating scales (e.g., Binary, 
Graphic-Precise, Graphic-Vague, Numeric-Precise, Numeric-Vague, Star-Numeric, and Star-Only) and found out that binary rating scales best help to counteract anchoring effects. For group recommender systems, Stettinger et al. [146] pointed out that the later the disclosure of group members' preferences, the higher the discussion intensity inside a group. This helps to counteract anchoring effects and to increase the quality of decision outcomes.

\subsection{Framing Effects}

Framing effects are often detected in single-user recommendation scenarios, where a user's selection behavior is affected by how the decision-relevant information is presented [46]. These effects refer to alternative representations of the same objective information that significantly alter a user's assumptions, models, and ultimate decisions [64]. Framing effects can be explained by the prospect theory [81], indicating that users dislike losses more than they like equivalent gains. This means, when comparing the same amount of a potential loss and gain of an item, the loss has a higher emotional impact than the gain. In the existing literature, framing effects have also been investigated in group decisions [105]. Some examples thereof are References [88, 112], in which the findings are quite diverse. Kuhberger [88] found out that groups are usually less susceptible to framing effects than individuals. Paese et al. [112] show that impacts of framing effects on groups are different depending on how the frame is presented.

Application of framing effects: Similar to decoy and anchoring effects, framing effects can be exploited to increase the selection probability of recommended items. For instance, framing price and payment methods can trigger a potential focus shift from quality attributes to other item attributes (e.g., price) [46, 77]. A related example is the following: Two supermarkets (A and B) sell meat products. Supermarket $A$ offers $€ 21.50$ per $\mathrm{kg}$ of pork ribs with a $€ 1.25$ discount if the customer pays with cash, whereas supermarket $B$ offers $€ 20.25$ per $\mathrm{kg}$ of pork ribs with a surcharge of $€ 1.25$ if the customer pays with a credit card. In such a scenario, supermarket $A$ uses a positive framing, which suggests a potential gain. In contrast, supermarket $B$ uses a negative framing, which suggests a potential loss. Since customers are more likely to avoid losses, the offer of supermarket $A$ with a discount of $€ 1.25$ increases meat purchase, even though it is equivalent to the one offered by supermarket $B$. The mentioned framing type is a so-called attribute framing, which denotes the phenomenon that "different but equivalent" descriptions of decision alternatives can lead to different final decisions.

\subsection{Groupthink}

Groupthink is a cognitive bias triggered in group decisions, where group members focus on reaching a consensus rather than analyzing or considering existing conflicting preferences [48]. This bias becomes apparent if a group is highly cohesive, the leader insists on his favored solution, and the group is isolated from outside experts [96]. Groupthink can lead to sub-optimal decisions due to a lack of opposition and creativity. Furthermore, under groupthink situations, groups fail to analyze relevant alternatives in detail and do not focus on exchanging additional decision-relevant information [48]. This phenomenon increases the confirmation effect, indicating users' tendency to recall information units that confirm existing preferences [75]. Measures to counteract groupthink are the following: (i) the leader should not show his/her opinions to other group members during preference construction; (ii) external experts should participate in the group decision process and are encouraged to challenge group members' viewpoint; (iii) after achieving a consensus on open issues, an additional meeting can be triggered to help group members to discuss still-existing doubts and re-think the entire issue before coming to a final decision [76].

Application of Groupthink: Groupthink can be exploited in recommender systems to accelerate consensus-making processes. In scenarios where conflicts between group members' preferences 
are very high and consensus making lasts very long, groupthink becomes a benefit to help the group in establishing an agreement. In this scenario, a group leader's role can be utilized to achieve consensus, i.e., the leader's preference can be used as the standard to measure the consensus level among group members [167] (see also Section 5.7).

\section{OPEN RESEARCH ISSUES}

Although extensive research has already been performed to investigate the impacts of psychological factors on recommender systems, there are many open research issues to be solved within the scope of future work. In the following, we discuss these issues and possibilities to further integrate the discussed psychological factors into recommender systems.

\subsection{Personality}

In Section 3, we discussed personality-aware recommendation approaches that help to counteract challenges such as the new user cold-start problem and recommendation diversity. However, there are further possibilities how user personality can help to improve the recommendation quality.

7.1.1 Personality-based Cross-domain Recommendation. Although there exist some approaches (see References $[55,68]$ ) to personality-based cross-domain recommendation, insights into "how user personality can be used to better generate personalized recommendations" includes a couple of open issues. Indeed, the mentioned approaches were only tested in specific domains, such as movies, TV-shows, music, and books. It is questionable if these approaches are also helpful for other domains. Besides, we argue that with the same personality, a user might have different preferences for items stemming from different domains. For instance, a user's preferences for books and cameras could be different from each other. Thereby, using the user's preferences for books to infer his/her preferences for cameras could lead to imprecise recommendations. As a consequence, further related research is needed.

Personality-based cross-domain recommendations can also be extended and applied to group recommendation. One solution could be that the recommender system uses group members' personality traits to predict the favorite items of each individual in domain $A$ and domain $B$. Thereafter, an aggregated predictions approach [47] can be applied to merge the recommendations for individual group members and then to generate group recommendations.

7.1.2 Personality-based Explanations for Recommendations. Using users' personality helps to better detect users' preferences for items and provide more personalized recommendations. In this context, it is also essential to exploit personality characteristics for explanation purposes. An example of a personality-aware explanation is the following: "You are a reflective person who is quite open to experiences. Therefore, we recommend jazz and classical music to you" [128].

\subsection{Emotions}

The current literature (see Section 4) shows that emotions have a strong influence on how users choose items [94]. Vice versa, items can also trigger emotions, e.g., a user feels joy when listening to an excellent performance at a concert or feels sad when listening to the music of a late-night movie [90]. Although many studies on the usage of emotions in recommender systems have been performed, researchers primarily focus on the music and movie domains. Still, some open issues in these domains should be taken into account in future work. Some examples thereof are "How to precisely capture a user's emotions evoked when he/she is watching a movie or listening to a song?", "Which techniques can be applied to interpret the captured emotions?", and "How can emotion-based recommendation be combined with other recommendation techniques?" [14]. 
Besides the domains of music and movies, emotion-related factors could also be investigated in other domains such as retailing, commercials, tourism, or other domains where users' emotions strongly influence item consumption [14] (see also Section 4.2).

\subsection{Group Dynamics}

While considerable research has been conducted on how psychological factors affect decisionmaking processes, most of this research focuses on single-user recommendation scenarios. The integration of the mentioned factors in group decision-making processes is still under-explored. Particularly, the impacts of group composition features (e.g., group size, age, gender, culture, and group cohesiveness) on decision quality is not completely clear. It is still unclear in which way to connect fairness and consensus aspects with group composition features. Group members' fairness and consensus perceptions could differ depending on their age, gender, and culture. These factors might also be influenced by group size or group cohesiveness. In large groups, group members' preferences could be quite diverse. Thus, reaching consensus in such groups is more challenging than in small groups. To dive deeper into this topic, we will now discuss possibilities to further investigate the influences of group composition features on group decision making.

7.3.1 Group Size. Group size can directly affect the outcome of a group decision [40]. It is easier for small groups to reach a consensus than for large groups. In small groups, group members adequately express their opinions, listen to each other, and search for clarifications. On the contrary, in large groups, the probability of achieving a consensus is quite limited. The larger the group, the lower the cohesiveness and the higher the number of potential conflicts [139]. Further research on group decision-making behavior depending on different group sizes is needed to figure out "which group recommendation strategies can be used in which group size."

7.3.2 Age Diversity. Users of different ages do not show the same behavior when making a decision. For instance, young people are more likely to face pressure from emotional and social factors than adults and retired people [132]. The age difference among group members raises some open questions [158]: (1) "Do group decisions made by elderly users have an increased social awareness?" and (2) "Does the age diversity of users trigger more discussions to reach a consensus?"

7.3.3 Gender Diversity. Gender differences of group members can significantly influence the quality of group decisions [174]. Khasawneh and Abu-Shanab [84] conducted a user survey to measure the influence of group members' gender diversity on the performance of group decision making. Empirical results show that female-only groups perform better than male-only groups in terms of good ideas exchanged among group members. In the fund management domain, Borgan et al. [17] investigated the influence of gender diversity on the investment decision-making behavior of group members. The authors found out that a male presence in a team can increase the probability of choosing a higher risk investment and decrease the probability of choosing a loss investment. A related idea can be used to analyze the influence of gender diversity on group members' social awareness. For instance, a question that should be answered is: "Does gender diversity affect the fairness and consensus perceptions of group members concerning recommended items?"

7.3.4 Cultural Diversity. In the era of globalization, it is vital to understand the influences of national culture on multi-national projects and the performance of decision making. In multinational companies, there is a high probability of decision-making processes performed by group members with different cultural backgrounds. The cultural diversity of group members can affect decision-making styles, decision-making speed, the acceptance of changes, the willingness to make unfamiliar decisions, and the clearness of responsibilities [106]. Due to considerable impacts of cultural differences on group decision making, it makes sense to take into account social factors in 
groups together with cultural diversity. We assume that additionally considering cultural diversity in group decision making can help to better simulate group decisions and improve the quality of decision outcomes.

7.3.5 Group Cohesiveness. Group cohesiveness describes a social process where group members interact with each other and generate the forces that push group members closer together [118]. In group decision making, the cohesiveness of group members can influence how a group decision is made. Group members' social relations should be analyzed to further investigate the impact on group decision making. In particular, social relations have to be analyzed in different cohesiveness degrees ranging from high cohesiveness (e.g., groups of family members or longtime friends) to low cohesiveness (e.g., ad hoc groups). In this context, the following research questions are of relevance [157]: (1) "Do cohesive groups target supportive communications, in which users are more comfortable expressing their thoughts and feelings than non-cohesive groups?", (2) "Are users in cohesive groups more friendly and cooperative than in non-cohesive groups?", and (3) "Do users in cohesive groups show a higher level of social awareness (e.g., fairness and consensus perceptions) and satisfaction with recommended items compared to users in non-cohesive groups?"

\subsection{Decision Biases}

We have just discussed how decision biases can influence the decision-making behavior of users. Although a couple of research contributions provide analyses of decision biases in different domains, most of them were discussed in single-user recommendation contexts. There are still missing in-depth analyses of decision biases in group decision making. For instance, polarization effects can occur in group decisions, which lead to more polarized decisions compared to group members' initial preferences. Although some effects have already been detected in high-involvement item domains [9], it is still unclear in which scenarios the knowledge about these effects can be exploited to improve the quality of group decisions. Besides, further decision biases need to be investigated in group recommender systems. For instance, shared-information biases refer to a tendency in which groups only discuss the information available to all group members. These biases can prevent users from discovering hidden profiles [12]. In the context of group decision making, when evaluating items, group members tend to stick with the available information. Although this information helps users to save evaluation efforts, users' prior knowledge sometimes does not adequately reflect the decision situation. Consequently, this results in imprecise evaluations of items and inconsistencies among the preferences of group members. In this context, detecting biases and mitigating them are much-needed capabilities to improve decision quality. One solution to counteract these effects is to recommend dissenting items [135], which could increase knowledge exchange among group members. Such items can help to discover hidden profiles, which is the premise of generating high-quality group decisions [8]. Although the mentioned biases have already been investigated, insights on how to apply these in group recommender systems are still needed.

\section{CONCLUSION}

Knowledge about psychological factors in human decision making is extremely important to provide recommender systems with high-quality decision support. This article is a "full landscape" of existing research on the influence of psychological factors (e.g., personality, emotions, group dynamics, and decision biases) on recommender systems. We discussed the applications of user personality to (1) increase the prediction quality of recommender systems, (2) resolve the cold-start problem, and (3) diversify recommendations. We discussed the impacts of emotions on recommender systems and introduced an example of how to apply this aspect to boost the 
recommendation performance. Besides single-user recommendation scenarios, this article also discussed the impacts of psychological factors in group recommendation scenarios. Particularly, we analyzed existing studies on the influences of group dynamics such as social trust, emotional contagion, group personality composition, conformity, the role of group members, fairness, and consensus. Furthermore, to improve group recommendation quality, we presented novel recommendation approaches based on the preferences of group members and group dynamics. We summarized different types of decision biases, analyzed their impact on decision-making processes, and discussed their applications in the recommendation context. Although various studies have been performed in this line of research, plenty of open issues still exist.

\section{REFERENCES}

[1] H. Abdollahpouri, R. Burke, and B. Mobasher. 2017. Controlling popularity bias in learning-to-rank recommendation. In Proceedings of the 11th ACM Conference on Recommender Systems (RecSys'17). ACM, New York, NY, 42-46.

[2] A. Abdul, J. Chen, H. Liao, and S. Chang. 2018. An emotion-aware personalized music recommendation system using a convolutional neural networks approach. Appl. Sci. 8 (07 2018), 1103.

[3] G. Adomavicius, J. Bockstedt, C. Shawn, and J. Zhang. 2014. De-biasing user preference ratings in recommender systems. CEUR Workshop Proceedings 1253 (2014), 2-9.

[4] G. Adomavicius, J. C. Bockstedt, S. P. Curley, and J. Zhang. 2013. Do recommender systems manipulate consumer preferences? A study of anchoring effects. Inf. Syst. Res. 24, 4 (22 Oct. 2013), 956-975.

[5] G. Adomavicius and A. Tuzhilin. 2005. Toward the next generation of recommender systems: A survey of the stateof-the-art and possible extensions. IEEE Trans. Knowl. Data Eng. 17, 6 (June 2005), 734-749.

[6] S. Amer-Yahia, S. B. Roy, A. Chawlat, G. Das, and C. Yu. 2009. Group recommendation: Semantics and efficiency. Proc. VLDB Endow. 2, 1 (Aug. 2009), 754-765.

[7] L. Ardissono, A. Goy, G. Petrone, M. Segnan, and P. Torasso. 2002. Tailoring the recommendation of tourist information to heterogeneous user groups. In Revised Papers from the International Workshops OHS-7, SC-3, and AH-3 on Hypermedia: Openness, Structural Awareness, and Adaptivity. Springer-Verlag, Berlin, 280-295.

[8] M. Atas, A. Felfernig, M. Stettinger, and T. N. T. Tran. 2017. Beyond item recommendation: Using recommendations to stimulate knowledge sharing in group decisions. In Social Informatics, G. L. Ciampaglia, A. Mashhadi, and T. Yasseri (Eds.). Springer International Publishing, Oxford, UK, 368-377.

[9] M. Atas, S. Reiterer, A. Felfernig, T. N. T. Tran, and M. Stettinger. 2018. Polarization effects in group decisions. In Proceedings of the 26th Conference on User Modeling, Adaptation and Personalization (UMAP'18). ACM, New York, NY, 305-310.

[10] M. Atas, T. N. T. Tran, R. Samer, A. Felfernig, M. Stettinger, and D. Fucci. 2018. Liquid democracy in group-based configuration. In Proceedings of the 20th Configuration Workshop, Graz, Austria, September 27-28, 2018. CEUR. 93-98.

[11] Y. Bachrach, M. Kosinski, T. Graepel, P. Kohli, and D. Stillwell. 2012. Personality and patterns of Facebook usage. In Proceedings of the 4th ACM Web Science Conference (WebSci'12). Association for Computing Machinery, New York, NY, 24-32.

[12] D. F. Baker. 2010. Enhancing group decision making: An exercise to reduce shared information bias. F. Manag. Educ. 34, 2 (2010), 249-279.

[13] L. Baltrunas and F. Ricci. 2014. Experimental evaluation of context-dependent collaborative filtering using item splitting. User Model. User-adapt. Interact. 24, 1-2 (Feb. 2014), 7-34.

[14] S. Berkovsky. 2015. Emotion-based movie recommendations: How far can we take this? In Proceedings of the 3rd Workshop on Emotions and Personality in Personalized Systems (EMPIRE'15). ACM, New York, NY, 1-1.

[15] S. Berkovsky and J. Freyne. 2010. Group-based recipe recommendations: Analysis of data aggregation strategies. In Proceedings of the 4th ACM Conference on Recommender Systems (RecSys'10). ACM, New York, NY, 111-118.

[16] C. Bologna, A. C. De Rosa, A. De Vivo, Matteo Gaeta, Giuseppe Sansonetti, and V. Viserta. 2013. Personality-based recommendation in E-commerce. CEUR Workshop Proceedings 997 (2013), 1-6.

[17] V. L. Borgan, D. R. Just, and C. S. Dev. 2012. Team gender diversity and investment decision making behavior. Rev. Behav. Fin. 5, 2 (2012), 134-152.

[18] M. M. Bradley and P. J. Lang. 1994. Measuring emotion: The self-assessment manikin and the semantic differential. 7. Behav. Therap. Exper. Psychiat. 25, 1 (1994), 49-59.

[19] S. J. Brams. 2012. Fair Division. Springer New York, New York, NY, 1073-1080.

[20] R. Brown. 1988. Group Processes. John Wiley and Sons Ltd, Oxford, UK.

[21] R. Burke. 2002. Hybrid recommender systems: Survey and experiments. User Model. User-adapt. Interact. 12, 4 (Nov. 2002), 331-370

[22] R. Burke, A. Felfernig, and M. H. Göker. 2011. Recommender systems: An overview. AI Mag. 32 (2011), 13-18. 
[23] I. Cantador and I. Fernández-Tobías. 2014. On the exploitation of user personality in recommender systems. In Proceedings of the 1st International Workshop on Decision Making and Recommender Systems (DMRS'14). 42-45.

[24] I. Cantador, I. Fernández-Tobías, and R. Bellogín. 2013. Relating personality types with user preferences in multiple entertainment domains. CEUR Workshop Proceedings 997 (2013), 1-17.

[25] D. Cao, X. He, L. Miao, Y. An, C. Yang, and R. Hong. 2018. Attentive group recommendation. In Proceedings of the 41st International ACM SIGIR Conference on Research \& Development in Information Retrieval (SIGIR'18). Association for Computing Machinery, New York, NY, 645-654.

[26] L. A. M. C. Carvalho and H. T. Macedo. 2013. Users' satisfaction in recommendation systems for groups: An approach based on noncooperative games. In Proceedings of the 22nd International Conference on World Wide Web (WWW'13). ACM, New York, NY, 951-958.

[27] G. Castellano, L. Kessous, and G. Caridakis. 2008. Emotion Recognition Through Multiple Modalities: Face, Body Gesture, Speech. Springer Berlin, 92-103.

[28] J. Castro, F. J. Quesada, I. Palomares, and L. Martínez. 2015. A consensus-driven group recommender system. Int. F. Intell. Syst. 30, 8 (2015), 887-906.

[29] G. Charness and M. Rabin. 2002. Understanding social preferences with simple test. Quart. f. Econ. 117, 3 (08 2002), 817-869.

[30] L. Chen, M. de Gemmis, A. Felfernig, P. Lops, F. Ricci, and G. Semeraro. 2013. Human decision making and recommender systems. ACM Trans. Interact. Intell. Syst. 3, 3 (Oct. 2013).

[31] L. Chen and P. Pu. 2005. Trust building in recommender agents. In Proceedings of the 1st International Workshop on Web Personalization, Recommender Systems and Intelligent User Interfaces (WPRSIUI'05). Word Scientific, London, 135-145.

[32] L. Chen, W. Wu, and L. He. 2013. How personality influences users' needs for recommendation diversity? In CHI'13 Extended Abstracts on Human Factors in Computing Systems (CHI EA'13). ACM, New York, NY, 829-834.

[33] Y. Chen and P. Pu. 2012. CoFeel: Using emotions for social interaction in group recommender systems. CEUR Workshop Proceedings 891 (01 2012), 48-55.

[34] Y. Chen and P. Pu. 2014. Designing emotion awareness interface for group recommender systems. In Proceedings of the International Working Conference on Advanced Visual Interfaces (AVI'14). ACM, New York, NY, 347-348.

[35] I. A. Christensen and S. Schiaffino. 2014. Social influence in group recommender systems. Online Inf. Rev. 38, 4 (2014), 524-542.

[36] P. Cremonesi, A. Donatacci, F. Garzotto, and R. Turrin. 2012. Decision-making in recommender systems: The role of user's goals and bounded resources. In Decisions@RecSys (CEUR Workshop Proceedings), M. de Gemmis, A. Felfernig, P. Lops, F. Ricci, G. Semeraro, and M. C. Willemsen (Eds.), Vol. 893. CEUR-WS.org, 1-7.

[37] A. Delic, J. Masthoff, J. Neidhardt, and H. Werthner. 2018. How to use social relationships in group recommenders: Empirical evidence. In Proceedings of the 26th Conference on User Modeling, Adaptation and Personalization (UMAP’18), T. Mitrovic, L. Zhang, L. Chen, and D. Chin (Eds.). ACM, New York, NY, 121-129.

[38] A. Delic, J. Neidhardt, T. N. Nguyen, F. Ricci, L. Rook, H. Werthner, and M. Zanker. 2016. Observing group decision making processes. In Proceedings of the 10th ACM Conference on Recommender Systems (RecSys'16). Association for Computing Machinery, New York, NY, 147-150.

[39] A. Delic, F. Ricci, and J. Neidhardt. 2019. Preference networks and non-linear preferences in group recommendations. In Proceedings of the IEEE/WIC/ACM International Conference on Web Intelligence (WI'19), P. M. Barnaghi, G. Gottlob, Y. Manolopoulos, T. Tzouramanis, and A. Vakali (Eds.). ACM, New York, NY, 403-407.

[40] G. Desanctis and R. B. Gallupe. 1987. A foundation for the study of group decision support systems. Manag. Sci. 33, 5 (May 1987), 589-609.

[41] G. Dunn, J. Wiersema, J. Ham, and L. Aroyo. 2009. Evaluating interface variants on personality acquisition for recommender systems. In User Modeling, Adaptation, and Personalization, G. J. Houben, G. McCalla, F. Pianesi, and M. Zancanaro (Eds.). Springer Berlin, 259-270.

[42] P. Ekman. 1999. Basic Emotions, Handbook of Cognition and Emotion. John Wiley \& Sons, Ltd., Sussex, Chichester, West Sussex, UK.

[43] M. Elahi, M. Braunhofer, F. Ricci, and M. Tkalčič. 2013. Personality-based active learning for collaborative filtering recommender systems. In Proceedings of the 13th International Conference on Advances in Artificial Intelligence, Vol. 8249. Springer-Verlag New York, Inc., New York, NY, 360-371.

[44] G. Fang, L. Su, D. Jiang, and L. Wu. 2018. Group recommendation systems based on external social-trust networks. Wirel. Commun. Mob. Comput. 2018 (05 2018), 1-11.

[45] G. Farnadi, G. Sitaraman, S. Sushmita, F. Celli, M. Kosinski, D. Stillwell, S. Davalos, M. Moens, and M. De Cock. 2016. Computational personality recognition in social media. User Model. User-adapt. Interact. 26, 2-3 (2016), 109-142.

[46] A. Felfernig. 2014. Biases in decision making. In Proceedings of the International Workshop on Decision Making and Recommender Systems 2014. CEUR, 32-37. 
[47] A. Felfernig, M. Atas, D. Helic, T. N. T. Tran, M. Stettinger, and R. Samer. 2018. Group Recommender Systems: An Introduction. Springer, NY, 27-58.

[48] A. Felfernig, M. Atas, M. Stettinger, T. N. T. Tran, and G. Leitner. 2018. Group Recommender Systems: An Introduction. Springer, NY, 145-155.

[49] A. Felfernig, M. Atas, T. N. T. Tran, M. Stettinger, S. P. Erdeniz, and G. Leitner. 2017. An analysis of group recommendation heuristics for high- and low-involvement items. In Advances in Artificial Intelligence: From Theory to Practice, S. Benferhat, K. Tabia, and M. Ali (Eds.). Springer International Publishing, 335-344.

[50] A. Felfernig, L. Boratto, M. Stettinger, and M. Tkalčič. 2018. Group Recommender Systems: An Introduction. Springer International Publishing, NY, 157-167.

[51] A. Felfernig, L. Boratto, M. Stettinger, and M. Tkalčč ‘ 2018. Group Recommender Systems: An Introduction. Springer, NY.

[52] A. Felfernig, G. Friedrich, B. Gula, M. Hitz, T. Kruggel, G. Leitner, R. Melcher, D. Riepan, S. Strauss, E. Teppan, and O. Vitouch. 2007. Persuasive recommendation: Serial position effects in knowledge-based recommender systems. In Persuasive Technology, Y. de Kort, W. IJsselsteijn, C. Midden, B. Eggen, and B. J. Fogg (Eds.). Springer Berlin, 283-294.

[53] A. Felfernig, B. Gula, G. Leitner, M. Maier, and R. Melcher. 2008. A dominance model for the calculation of decoy products in recommendation environments. In Proceedings of the AISB Symposium on Persuasive Technologies. The Society for the Study of Artificial Intelligence and Simulation of Behaviour, 43-50.

[54] A. Felfernig, B. Gula, and E. Teppan. 2007. Knowledge-based recommender technologies for marketing and sales. Int. 7. Pattern Recog. Artif. Intell. 21, 2 (2007), 333-354.

[55] I. Fernández-Tobías, M. Braunhofer, M. Elahi, F. Ricci, and I. Cantador. 2016. Alleviating the new user problem in collaborative filtering by exploiting personality information. User Model. User-adapt. Interact. 26, 2 (01 June 2016), 221-255.

[56] B. Ferwerda and M. Schedl. 2016. Personality-based user modeling for music recommender systems. In Proceedings of the International Conference on Machine Learning and Knowledge Discovery in Databases. Springer International Publishing, 254-257.

[57] D. Forsyth. 2006. Group Dynamics (7th ed.). Cengage, Boston, MA.

[58] M. Gartrell, X. Xing, Q. Lv, A. Beach, R. Han, S. Mishra, and K. Seada. 2010. Enhancing group recommendation by incorporating social relationship interactions. In Proceedings of the 16th ACM International Conference on Supporting Group Work (GROUP'10). ACM, New York, NY, 97-106.

[59] J. Golbeck. 2006. Combining provenance with trust in social networks for semantic web content filtering. In Provenance and Annotation of Data. Springer Berlin, 101-108.

[60] J. Golbeck. 2006. Generating predictive movie recommendations from trust in social networks. In Proceedings of the 4th International Conference on Trust Management (iTrust'06). Springer-Verlag, Berlin, 93-104.

[61] G. Gonzalez, J. L. de la Rosa, M. Montaner, and S. Delfin. 2007. Embedding emotional context in recommender systems. In Proceedings of the IEEE 23rd International Conference on Data Engineering Workshop. IEEE Service Center, 845-852.

[62] S. D. Gosling, P. J. Rentfrow, and W. B. Swann. 2003. A very brief measure of the Big-Five personality domains. F. Res. Personal. 37, 6 (2003), 504-528.

[63] M. P. Graus and B. Ferwerda. 23 Sep. 2019. Personalized Human-computer Interaction. De Gruyter Oldenbourg, Berlin, Boston, 1-30.

[64] D. J. Hardisty, E. J. Johnson, and E. U. Weber. 2010. A dirty word or a dirty world? Attribute framing, political affiliation, and query theory. Psychol. Sci. 21, 1 (2010), 86-92.

[65] S. Hareli and A. Rafaeli. 2008. Emotion cycles: On the social influence of emotion in organizations. Res. Organiz. Behav. 28 (2008), 35-59.

[66] S. Highhouse and A. Gallo. 1997. Order effects in personnel decision making. Hum. Perform. 10, 1 (1997), $31-46$.

[67] J. L. Holland. 1985. Making Vocational Choices: A Theory of Vocational Personalities and Work Environments. PrenticeHall, New Jersey. 84006796

[68] R. Hu. 2010. Design and user issues in personality-based recommender systems. In Proceedings of the 4th ACM Conference on Recommender Systems (RecSys'10). ACM, New York, NY, 357-360.

[69] R. Hu and P. Pu. 2010. A study on user perception of personality-based recommender systems. In User Modeling, Adaptation, and Personalization. Springer Berlin, 291-302.

[70] R. Hu and P. Pu. 2011. Enhancing collaborative filtering systems with personality information. In Proceedings of the 5th ACM Conference on Recommender Systems (RecSys'11). ACM, New York, NY, 197-204

[71] Y. Hu, Y. Koren, and C. Volinsky. 2008. Collaborative filtering for implicit feedback datasets. In Proceedings of the 2008 8th IEEE International Conference on Data Mining (ICDM'08). IEEE Computer Society, Washington, DC, 263-272.

[72] U. A. P. Ishanka and T. Yukawa. 2017. The prefiltering techniques in emotion based place recommendation derived by user reviews. Appl. Comput. Intell. Soft Comput. 2017 (2017), 1-10. 
[73] C. E. Izard. 1977. The Emotions. Plenum Press, New York, NY.

[74] A. Jameson, M. C. Willemsen, A. Felfernig, M. de Gemmis, P. Lops, G. Semeraro, and L. Chen. 2015. Human Decision Making and Recommender Systems. Springer US, Boston, MA, 611-648.

[75] I. L. Janis. 1972. Victims of groupthink: A psychological study of foreign-policy decisions and fiascoes. F. Amer. Hist. 60, 3 (1972), 857-858.

[76] I. L. Janis. 1982. Groupthink: Psychological Studies of Policy Decisions and Fiascoes. Houghton Mifflin, Boston, MA. 82015813

[77] D. Jannach, M. Zanker, A. Felfernig, and G. Friedrich. 2010. Recommender Systems an Introduction. Cambridge University Press, Cambridge, UK.

[78] R. L. Jenkins. 1978. Family vacation decision-making. F. Trav. Res. 16, 4 (Apr. 1978), 2-7.

[79] O. John and P. Srivastava. 1999. The Big Five Trait Taxonomy: History, Measurement, and Theoretical Perspectives. The Guilford Press, New York, NY, 102-138.

[80] P. Juslin. 2013. What does music express? Basic emotions and beyond. Front. Psychol. 4 (Sep. 2013), 1-14.

[81] D. Kahneman and A. Tversky. 1997. Prospect theory: An analysis of decision under risk. Econometrica 47, 2 (Mar. 1997), 263-291.

[82] T. Kameda, Y. Ohtsubo, and M. Takezawa. 1997. Centrality in sociocognitive networks and social influence: An illustration in a group decision-making context. J. Personal. Soc. Psychol. 73 (08 1997), 296-309.

[83] M. Kaminskas and F. Ricci. 2011. Location-adapted music recommendation using tags. In User Modeling, Adaption and Personalization, J. A. Konstan, R. Conejo, J. L. Marzo, and N. Oliver (Eds.). Springer Berlin, 183-194.

[84] R. T. Khasawneh and E. A. Abu-Shanab. 2013. Factors influencing group decision making performance in a GSS enabled environment. Comput. Sci. Inf. Technol. 1 (2013), 145-152.

[85] R. H. Kilmann and K. W. Thomas. 1977. Developing a forced-choice measure of conflict-handling behavior: The "mode" instrument. Educ. Psychol. Meas. 37, 2 (1977), 309-325.

[86] S. Köcher, M. Jugovac, D. Jannach, and H. H. Holzmüller. 2019. New hidden persuaders: An investigation of attributelevel anchoring effects of product recommendations. F. Retail. 95, 1 (2019), 24-41.

[87] M. Kosinski, D. Stillwell, and T. Graepel. 2013. Private traits and attributes are predictable from digital records of human behavior. Proc. Nat. Acad. Sci. 110, 15 (Mar. 2013), 5802-5805.

[88] A. Kühberger. 1998. The influence of framing on risky decisions: A meta-analysis. Organiz. Behav. Hum. Decis. Proc. 75, 1 (1 7 1998), 23-55.

[89] M. Kunaver and T. Pozr̆l. 2017. Diversity in recommender systems-A survey. Knowl.-based Syst. 123, c (May 2017), 154-162.

[90] F. F. Kuo, M. F. Chiang, M. K. Shan, and S. Y. Lee. 2005. Emotion-based music recommendation by association discovery from film music. In Proceedings of the 13th ACM International Conference on Multimedia (MULTIMEDIA'05). ACM, New York, NY, 507-510.

[91] B. Latané and S. Wolf. 1981. The social impact of majorities and minorities. Psychol. Rev. 88, 5 (1981), 438-453.

[92] Y. Lee and J. Jeong. 2004. A study on the analysis of emotion-expressing vocabulary for realtime conversion of Avatar's counternance. Korean Soc. Des. Sci.ence 56 (2004), 199-208.

[93] C. Lin and D. McLeod. 2002. Exploiting and learning human temperaments for customized information recommendation. In Proceedings of the 6th IASTED International Conference on Internet and Multimedia Systems and Applications. ACTA Press, 218-223.

[94] G. Loewenstein and J. S. Lerner. 2003. The Role of Affect in Decision Making. Oxford University Press, Oxford, UK, 619-642.

[95] F. Lu and N. Tintarev. 2018. A diversity adjusting strategy with personality for music recommendation. In Proceedings of the 5th foint Workshop on Interfaces and Human Decision Making for Recommender Systems (IntRS'18), co-located with ACM Conference on Recommender Systems (RecSys'18). ACM, 7-14.

[96] F. C. Lunenburg. 2010. Group decision making: The potential for groupthink. Int. f. Manag., Bus., Admin. 13, 1 (2010), $1-6$.

[97] M. Mandl, A. Felfernig, and M. Schubert. 2009. Consumer decision making in knowledge-based recommendation. In Active Media Technology, J. Liu, J. Wu, Y. Yao, and T. Nishida (Eds.). Springer Berlin, 69-80.

[98] M. Mandl, A. Felfernig, E. Teppan, and M. Schubert. 2011. Consumer decision making in knowledge-based recommendation. F. Intell. Inf. Syst. 37, 1 (01 Aug. 2011), 1-22.

[99] B. Marcus, F. Machilek, and A. Schütz. 2006. Personality in cyberspace: Personal Web sites as media for personality expressions and impressions. f. Personal. Soc. Psychol. 90, 6 (2006), 1014-1031.

[100] D. Markovikj, S. Gievska, M. Kosinski, and D. Stillwell. 2013. Mining Facebook data for predictive personality modeling. In Proceedings of the 7th International AAAI Conference on Weblogs and Social Media (ICWSM'13). The AAAI Press, Palo Alto, CA, 23-26.

[101] J. Masthoff. 2011. Group Recommender Systems: Combining Individual Models. Springer, New York, NY, 677-702. 
[102] J. Masthoff and A. Gatt. 2006. In pursuit of satisfaction and the prevention of embarrassment: Affective state in group recommender systems. User Model. User-adapt. Interact. 16, 3-4 (9 2006), 281-319.

[103] R. R. Mccrae and O. P. John. 1992. An introduction to the five-factor model and its applications. F. Personal. 60 (1992), $175-215$

[104] X. Meng, S. Wang, H. Liu, and Y. Zhang. 2018. Exploiting emotion on reviews for recommender systems. In Proceedings of the 32nd AAAI Conference on Artificial Intelligence (AAAI'18). AAAI Press, 3788-3795.

[105] K. F. Milch, E. U. Weber, K. C. Appelt, M. J. J. Handgraaf, and D. H. Krantz. 2009. From individual preference construction to group decisions: Framing effects and group processes. Organiz. Behav. Hum. Decis. Proc. 108, 2 (Mar. 2009), 242-255.

[106] R. Müller, K. Spang, and S. Ozcan. 2009. Cultural differences in decision making in project teams. Int. F. Manag. Projects Bus. 2, 1 (2009), 70-93.

[107] G. Nave, J. Minxha, D. M. Greenberg, M. Kosinski, D. Stillwell, and J. Rentfrow. 2018. Musical preferences predict personality: Evidence from active listening and Facebook likes. Psychol. Sci. 29, 7 (2018), 1145-1158.

[108] T. N. Nguyen and F. Ricci. 2017. Combining long-term and discussion-generated preferences in group recommendations. In Proceedings of the 25th Conference on User Modeling, Adaptation and Personalization (UMAP'17). ACM, New York, NY, 377-378.

[109] T. N. Nguyen, F. Ricci, A. Delic, and D. G. Bridge. 2019. Conflict resolution in group decision making: Insights from a simulation study. User Model. User-adapt. Interact. 29, 5 (2019), 895-941.

[110] M. A. S. N. Nunes and R. Hu. 2012. Personality-based recommender systems: An overview. In Proceedings of the 6th ACM Conference on Recommender Systems (RecSys'12). ACM, New York, NY, 5-6.

[111] M. O’Connor, D. Cosley, J. A. Konstan, and J. Riedl. 2001. PolyLens: A recommender system for groups of users. In Proceedings of the 7th Conference on European Conference on Computer Supported Cooperative Work (ECSCW'01). Kluwer Academic Publishers, Norwell, MA, 199-218.

[112] P. W. Paese, M. Bieser, and M. E. Tubbs. 1993. Framing effects and choice shifts in group decision making. Organiz. Behav. Hum. Decis. Proc. 56, 1 (1993), 149-165.

[113] I. Palomares, L. Martínez-López, and F. Herrera. 2014. A consensus model to detect and manage non-cooperative behaviors in large-scale group decision making. IEEE Trans. Fuzzy Syst. 22, 3 (2014), 516-530.

[114] J. Y. Pan, H. J. Yang, C. Faloutsos, and P. Duygulu. 2004. Automatic multimedia cross-modal correlation discovery. In Proceedings of the 10th ACM SIGKDD International Conference on Knowledge Discovery and Data Mining (KDD'04). Association for Computing Machinery, New York, NY, 653-658.

[115] M. J. Pazzani. 1999. A framework for collaborative, content-based and demographic filtering. Artif. Intell. Rev. 13, 5-6 (Dec. 1999), 393-408.

[116] I. J. Pérez, F. J. Cabrerizo, S. Alonso, Y. C. Dong, F. Chiclana, and E. Herrera-Viedma. 2018. On dynamic consensus processes in group decision making problems. Inf. Sci. 459 (May 2018), 20-35.

[117] J. Pincay, L. Terán, and E. Portmann. 2019. Health recommender systems: A state-of-the-art review. In Proceedings of the 6th International Conference on eDemocracy eGovernment (ICEDEG'19). IEEE, 47-55.

[118] W. E. Piper, M. Marrache, R. Lacroix, A. M. Richardsen, and B. D. Jones. 1983. Cohesion as a basic bond in groups. Hum. Relat. 36, 2 (1983), 93-108.

[119] M. Polignano. 2015. The influence of user's emotions in recommender systems for decision making processes. In Proceedings of the CHItaly 2015 Doctoral Consortium co-located with the 11th International Conference of the Italian SIGCHI Chapter (CHItaly 2015). CEUR Workshop Proceedings, 58-66.

[120] D. Quercia, M. Kosinski, D. Stillwell, and J. Crowcroft. 2011. Our Twitter profiles, our selves: Predicting personality with Twitter. In Proceedings of the PASSAT/SocialCom 2011, Privacy, Security, Risk and Trust (PASSAT), 2011 IEEE Third International Conference on and 2011 IEEE Third International Conference on Social Computing (SocialCom'2011). IEEE Computer Society, Boston, MA, 180-185.

[121] D. Quercia, R. Lambiotte, D. Stillwell, M. Kosinski, and J. Crowcroft. 2012. The personality of popular Facebook users. In Proceedings of the ACM Conference on Computer Supported Cooperative Work (CSCW'12). ACM, New York, NY, 955-964.

[122] L. Quijano-Sanchez, J. A. Recio-Garcia, and B. Diaz-Agudo. 2010. Personality and social trust in group recommendations. In Proceedings of the 22nd IEEE International Conference on Tools with Artificial Intelligence (ICTAI'10), Vol. 02. IEEE Computer Society, Washington, DC, 121-126.

[123] L. Quijano-Sanchez, J. A. Recio-Garcia, B. Diaz-Agudo, and G. Jimenez-Diaz. 2013. Social factors in group recommender systems. ACM Trans. Intell. Syst. Technol. 4, 1 (Feb. 2013).

[124] L. Quijano-Sanchez, C. Sauer, J. A. Recio-Garcia, and B. Diaz-Agudo. 2017. Make it personal: A social explanation system applied to group recommendations. Exp. Syst. Applic. 76 (2017), 36-48.

[125] E. Quintarelli, E. Rabosio, and L. Tanca. 2016. Recommending new items to ephemeral groups using contextual user influence. In Proceedings of the 10th ACM Conference on Recommender Systems (RecSys'16). ACM, New York, NY, $285-292$. 
[126] V. J. Ramos. 2018. Analyzing the Role of Cognitive Biases in the Decision-making Process. IGI Global, Hershey, PA.

[127] J. A. Recio-Garcia, G. Jimenez-Diaz, A. A. Sanchez-Ruiz, and B. Diaz-Agudo. 2009. Personality aware recommendations to groups. In Proceedings of the 3rd ACM Conference on Recommender Systems (RecSys'09). ACM, New York, NY, 325-328.

[128] P. J. Rentfrow and S. D. Gosling. 2003. The do re mi's of everyday life: The structure and personality correlates of music preferences. F. Personal. Soc. Psychol. 84, 6 (2003), 1236-1256.

[129] S. Rossi and F. Cervone. 2016. Social utilities and personality traits for group recommendation: A pilot user study. In Proceedings of the 8th International Conference on Agents and Artificial Intelligence (ICAART 2016), Volume 1, $\mathrm{H}$. Jaap van den Herik and Joaquim Filipe (Eds.). SciTePress, 38-46.

[130] J. A. Russell. 1980. A circumplex model of affect. F. Personal. Soc. Psychol. 39, 6 (1980), 1161-1178.

[131] C. Sammut and G. I. Webb. 2010. TF-IDF. Springer US, Boston, MA, 986-987.

[132] M. L. Sanz de Acedo Lizárrage, M. T. Sanz de Acedo Baquedano, and María Cardelle-Elawar. 2007. Factors that affect decision making: Gender and age differences.Int. f. Psychol. Psycholog. Therap.y 7, 3 (2007), 381-391.

[133] D. L. Schacter, D. T. Gilbert, and D. M. Wegner. 2010. Psychology. Worth Publishers, NY. 2010940234

[134] U. Schimmack and A. Grob. 2000. Dimensional models of core affect: A quantitative comparison by means of structural equation modeling. Eur. F. Personal. 14, 4 (07 2000), 325-345.

[135] C. Schwind and J. Buder. 2012. Reducing confirmation bias and evaluation bias: When are preference-inconsistent recommendations effective-And when not? Comput. Hum. Behav. 28, 6 (2012), 2280-2290.

[136] D. Serbos, S. Qi, N. Mamoulis, E. Pitoura, and P. Tsaparas. 2017. Fairness in package-to-group recommendations. In Proceedings of the 26th International Conference on World Wide Web (WWW'17). International World Wide Web Conferences Steering Committee, 371-379.

[137] M. K. Shan, F. F. Kuo, M. F. Chiang, and S. Y. Lee. 2009. Emotion-based music recommendation by affinity discovery from film music. Exp. Syst. Applic. 36, 4 (2009), 7666-7674.

[138] H. Sharp and P. Mott. 1956. Consumer decisions in the metropolitan family. f. Market. 21, 2 (Oct. 1956), 149-156.

[139] M. E. Shaw. 1976. Group Dynamics: The Psychology of Small Group Behavior. McGraw-Hill, NY.

[140] L. Shu, J. Xie, M. Yang, Z. Li, Z. Li, D. Liao, X. Xu, and X. Yang. 2018. A review of emotion recognition using physiological signals. Sensors 18, 7 (2018), 1-41.

[141] G. B. Sigal. 2002. The ripple effect: Emotional contagion and its influence on group behavior. Admin. Sci. Quart. 47, 4 (2002), 644-675.

[142] M. Song, H. Namgoong, H. G. Kim, and J. Eune. 2009. A proposed movie recommendation method using emotional word selection. In Online Communities and Social Computing. Springer Berlin, 525-534.

[143] K. E. Stanovich and R. F. West. 2008. On the relative independence of thinking biases and cognitive ability. F. Personal. Soc. Psychol. 94, 4 (2008), 672-695.

[144] B. Stark, C. Knahl, M. Aydin, and K. Elish. 2019. A literature review on medicine recommender systems. International 7. Adv. Comput. Sci. Applic. 10, 8 (2019), 6-13.

[145] M. Stettinger. 2014. Choicla: Towards domain-independent decision support for groups of users. In Proceedings of the 8th ACM Conference on Recommender Systems (RecSys'14). ACM, 425-428.

[146] M. Stettinger, A. Felfernig, G. Leitner, and S. Reiterer. 2015. Counteracting anchoring effects in group decision making. In Proceedings of the 23rd Conference on User Modeling, Adaptation and Personalization (UMAP'15) (Lecture Notes in Computer Science), Vol. 9146. Springer, Trinity College, 118-130.

[147] M. Stettinger, A. Felfernig, G. Leitner, S. Reiterer, and M. Jeran. 2015. Counteracting serial position effects in the CHOICLA group decision support environment. In Proceedings of the 20th International Conference on Intelligent User Interfaces (IUI'15). Association for Computing Machinery, New York, NY, 148-157.

[148] M. Stettinger, T. N. T. Tran, I. Pribik, G. Leitner, A. Felfernig, R. Samer, M. Atas, and M. Wundara. 2020. KNowLEDGECHECKR: Intelligent techniques for counteracting forgetting. In Proceedings of 24th European Conference on Artificial Intelligence (PAIS ECAI'20). IOS Press.

[149] H. Tajfel. 2010. Social Identity and Intergroup Relations. Cambridge University Press, Cambridge, UK.

[150] M. Tamir. 2005. Don't worry, be happy? Neuroticism, trait-consistent affect regulation, and performance. F. Personal. Soc. Psychol. 89, 3 (2005), 449-461.

[151] E. Teppan and A. Felfernig. 2009. The asymmetric dominance effect and its role in e-tourism recommender applications. In Proceedings of the Wirtschaftsinformatik (WI'2009). Austrian Computer Society (OCG), 791-800.

[152] E. Teppan and A. Felfernig. 2012. Minimization of decoy effects in recommender result sets. Web Intell. Agent Syst. 10, 4 (2012), 385-395.

[153] E. Teppan, A. Felfernig, and K. Isak. 2011. Decoy effects in financial service E-sales systems. In Proceedings of the RecSys'11 Workshop on Human Decision Making in Recommender Systems (Decision@RecSys'11). ACM, 1-8.

[154] E. C. Teppan and M. Zanker. 2015. Decision biases in recommender systems. f. Internet Commerce 14, 2 (2015), 255-275. 
[155] K. W. Thomas. 1992. Conflict and conflict management: Reflections and update. F. Organiz. Behav. 13, 3 (1992), 265-274.

[156] K. W. Thomas and R. H. Kilmann. 1974. Thomas-Kilmann Conflict Mode Instrument. Consulting Psychologists Press.

[157] L. Thompson, L. J. Kray, and E. A. Lind. 1998. Cohesion and respect: An examination of group decision making in social and escalation dilemmas. f. Experim. Soc. Psychol. 34, 3 (1998), 289-311.

[158] P. R. Thornton, G. Shaw, and A. M. Williams. 1997. Tourist group holiday decision-making and behaviour: The influence of children. Tour. Manag. 18, 5 (1997), 287-297.

[159] N. Tintarev, M. Dennis, and J. Masthoff. 2013. Adapting recommendation diversity to openness to experience: A study of human behaviour. In User Modeling, Adaptation, and Personalization. Springer Berlin, 190-202.

[160] M. Tkalčic and L. Chen. 2015. Recommender Systems Handbook. Springer, New York, NY, 715-739.

[161] M. Tkalčič, B. De Carolis, M. De Gemmis, A. Odic, and A. Kosir. 2017. Introduction to emotions and personality in personalized systems. In Emotions and Personality in Personalized Services-Models, Evaluation and Applications, M. Tkalčič, B. De Carolis, M. De Gemmis, A. Odic, and A. Kosir (Eds.). Springer, NY, 3-11.

[162] M. Tkalčič, A. Košir, and J. Tasič. 2011. Affective recommender systems: The role of emotions in recommender systems. CEUR Workshop Proceedings 811 (01 2011).

[163] M. Tkalčič, M. Kunaver, J. Tasič, and A. Košir. 2009. Personality based user similarity measure for a collaborative recommender system. In Proceedings of the 5th Workshop on Emotion in Human Computer Interaction-Real World Challenges. Fraunhofer-Publica, Cambridge University, Cambridge, UK, 30-37.

[164] R. W. Toseland, L. V. Jones, and Z. D. Gellis. 2004. Group Dynamics. The Guilford Press, New York, 13-31.

[165] T. N. T. Tran, M. Atas, A. Felfernig, V. M. Le, R. Samer, and M. Stettinger. 2019. Towards social choice-based explanations in group recommender systems. In Proceedings of the 27th ACM Conference on User Modeling, Adaptation and Personalization (UMAP'19). ACM, New York, NY, 13-21.

[166] T. N. T. Tran, M. Atas, A. Felfernig, R. Samer, and M. Stettinger. 2018. Investigating serial position effects in sequential group decision making. In Proceedings of the 26th Conference on User Modeling, Adaptation and Personalization (UMAP’18). ACM, New York, NY, 239-243.

[167] V. Tundjungsari, J. E. Istiyanto, E. Winarko, and R. Wardoyo. 2012. Achieving consensus with individual centrality approach. CoRR abs/1203.5570 (2012).

[168] G. A. Van Kleef and A. H. Fischer. 2016. Emotional collectives: How groups shape emotions and emotions shape groups. Cog. Emot. 30, 1 (2016), 3-19.

[169] S. Wasserman and K. Faust. 1994. Social Network Analysis: Methods and Applications. Cambridge University Press, Cambridge, UK.

[170] H. C. Wu, R. W. P. Luk, K. F. Wong, and K. L. Kwok. 2008. Interpreting TF-IDF term weights as making relevance decisions. ACM Trans. Inf. Syst. 26, 3 (June 2008).

[171] W. Wu, L. Chen, and Y. Zhao. 2018. Personalizing recommendation diversity based on user personality. User Model. User-adapt. Interact. 28, 3 (Aug. 2018), 237-276.

[172] L. Xiao, Z. Min, Z. Yongfeng, G. Zhaoquan, L. Yiqun, and M. Shaoping. 2017. Fairness-aware group recommendation with Pareto-efficiency. In Proceedings of the 11th ACM Conference on Recommender Systems (RecSys'17). ACM, New York, NY, 107-115.

[173] L. Xiao and G. Zhaoquan. 2017. How does fairness matter in group recommendation. In Proceedings of the Conference on Web Information Systems Engineering (WISE'17). Springer International Publishing, 458-466.

[174] S. Zaidi, M. Saif, and A. Zaheer. 2010. The effect of workgroup heterogeneity on decision making: An empirical investigation. Afric. F. Bus. Manag. 4, 10 (2010), 2132-2139.

[175] Y. Zheng, B. Mobasher, and R. D. Burke. 2013. The role of emotions in context-aware recommendation. In Proceedings of the 3rd Workshop on Human Decision Making in Recommender Systems in Conjunction with the 7th ACM Conference on Recommender Systems (Decision@RecSys'13). ACM, 21-28.

Received June 2020; revised December 2020; accepted January 2021 